This work has been submitted to Journal of Climate. Copyright in this work may be transferred without further notice. Please note that the manuscript is currently under review and has yet to be formally accepted for publication. Subsequent versions of this manuscript may have slightly different content. If accepted, the final version of this manuscript will be available via the 'Peerreviewed Publication DOI' link on the right-hand side of this webpage. 


\title{
Drivers of Local Ocean Heat Content Variability in ECCOv4
}

\author{
Jan-Erik Tesdal* and Ryan P. Abernathey
}

Lamont-Doherty Earth Observatory, Columbia University, Palisades, New York, USA

${ }_{4}{ }^{*}$ Corresponding author: Jan-Erik Tesdal, tesdal@1deo.columbia.edu 
ABSTRACT

${ }_{5}$ Variation in upper ocean heat content is a critical factor in understanding global climate variability.

- By using temperature anomaly budgets in a physically consistent ocean state estimate we describe

, the balance between atmospheric forcing and ocean transport mechanisms for different depth

${ }_{8}$ horizons and at varying temporal and spatial resolutions. The processes controlling local variations

${ }_{9}$ in ocean heat content differ in relevance depending on region and time scale. The advection term

10 dominates in the tropical oceans, while forcing is most relevant at higher latitudes and in parts

${ }_{11}$ of the subtropics. When integrating over greater depths, the forcing signal clearly weakens and

${ }_{12}$ advective heat convergence becomes more dominant. Temporal aggregation shows that advection

${ }_{13}$ becomes the principal term that determines variability at longer timescales. Ocean heat variability

${ }_{14}$ is due to anomalies in circulation, while the effect of anomalies in temperature are constrained

${ }_{15}$ to specific regions and increase in relevance with temporal aggregation. Even though there is a

${ }_{16}$ shift in the relative importance of forcing and advection with coarser horizontal resolution, the

${ }_{17}$ overall balance between the budget terms is remarkably insensitive to the spatial scale. A novel

18 cluster analysis was used to identify regions with similar underlying mechanisms relevant to ocean

19 heat content variability. Advection-driven regions coincide with strong currents such as western

20 boundary currents, the Antarctic Circumpolar Current and the tropics, while regions with a strong

${ }_{21}$ forcing signal are defined by shallower wintertime mixed layers and weak velocity fields. The vast

${ }_{22}$ majority of the ocean includes significant contributions by both forcing and advection. 


\section{Introduction}

Earth's oceans play a critical role in regulating the global climate system (Bigg et al. 2003; von Schuckmann et al. 2016) and have been shown to act as a critical sink of excess atmospheric and land-based heat resulting from greenhouse gases (e.g., Barnett et al. 2001, 2005; Pierce et al. 2012; Trenberth et al. 2014). Heat is also redistributed within and released from the oceans, thereby impacting atmospheric temperatures and the global climate system (Bigg et al. 2003). Ocean heat redistribution determines how effectively oceans can store excess heat due to anthropogenic warming, and played a key role in the 1998-2012 global surface warming hiatus (Yan et al. 2016; Liu and Xie 2018). Therefore, a clear understanding of heat transport mechanisms should enable better predictions regarding the extent of global and regional climate change (Keenlyside et al. 2008; Robson et al. 2012; Roberts et al. 2016).

Since heat is conserved, one powerful approach for understanding ocean heat content (OHC) variability is via the ocean heat budget. The budget relates a change in $\mathrm{OHC}$ to a variety of driving mechanisms that appear in the heat conservation equation (1), such as advection, diffusion, and air-sea forcing. Better understanding of which terms in the heat budget matter most can help us interpret patterns of ocean warming and think about how they might change in the future. Evaluating ocean heat budgets from direct observations is very difficult, and some un-observable processes must inevitably be inferred from the residual of better-known terms (Roberts et al. 2017).

The recent emergence of conservative ocean reanalysis products which assimilate observations in a dynamically consistent way-such as the ECCOv4 product used here-offers an exciting new opportunity to examine the historical ocean heat budget in precise detail.

However, a significant downfall of the budget approach is its complexity. Depending on how it is constructed, the budget can contain up to a dozen different terms (Piecuch and Ponte 2012; 
Buckley et al. 2014, 2015; Piecuch et al. 2017). Furthermore, the budget is evidently quite sensitive to spatial and temporal scale, and different regions of the ocean may have qualitatively different heat budgets (Bishop et al. 2017; Small et al. 2019, 2020). From this complexity, it can be hard to discern general, robust properties of the ocean heat budget.

The goal of our paper is to combine three recent methodological advances to try to reach some robust conclusions about the global heat budget. These methodological advances are the following:

1. The development of data-constrained yet dynamically consistent ocean reanalyses, which provide a precise, numerically closed heat budget at each grid point (Forget et al. 2015).

2. The "covariance ratio" analysis technique, first developed by Doney et al. (2007) and further elaborated by Bishop et al. (2017); Small et al. $(2019,2020)$. This method reduces the full timeseries of heat budget terms at each point in space (or averaged over a region) to a concise set of non-dimensional $O(1)$ values characterizing the importance of each term.

3. Unsupervised machine learning, which can help reveal latent patterns in large datasets. Kmeans clustering (Hartigan and Wong 1979; Gong and Richman 1995; Lund and Li 2009) has been successfully applied in oceanography to a wide variety of categorization problems, from identifying regimes of Southern Ocean phytoplankton blooms (Ardyna et al. 2017) to the ocean vorticity budget (Sonnewald et al. 2019). Here we apply clustering to the covariance ratios to identify regions with similar heat content dynamics.

Along the way, we take great care to examine the sensitivity of our results to spatial and temporal scales, in order to determine which patterns are most robust across scales.

With this analysis, a key question we hope to answer is under what circumstances is OHC variability primarily driven by atmospheric variability vs. internal mechanisms? For the internal driving mechanisms, what is the relative importance of advection vs. diffusion? And for advection, 
what is the relative importance of variations in ocean currents vs. variations in temperature; and of horizontal vs. vertical advective transport?

These are not new questions of course. Many past studies have attempted to understand the drivers of OHC and SST variability in different regions. In a classic pioneering study, (Hasselmann 1976) used a stochastic model to describe the temporal relationship between SST and forcing (i.e., the lead-lag correlation between surface heat flux, SST and its tendency). A series of subsequent studies have suggested that for much of the extratropical regions of the global ocean, SST variability is primarily a function of atmospheric-driven surface heat flux (e.g., von Storch 2000; Wu et al. 2006).

As the spatial resolution of SST and surface heat flux datasets have improved, Bishop et al. (2017) revised the connection between forcing and SST and highlighted regions where ocean dynamics clearly dominate. These regions are delineated by the western boundary currents (WBCs) and the Antarctic Circumpolar Current (ACC). Similarly, Small et al. (2019) showed that latent heat flux is primarily driven by variability in SST (i.e., driven by ocean dynamics) over the eastern tropical Pacific and mid-latitude ocean frontal zones (which are associated with WBCs). The above studies described only variability at the sea surface, but similar conclusions can be made for the upper ocean as well, given that SST variability is connected to temperature within the mixed layer (Alexander and Deser 1995). Looking at the upper ocean to full-depth OHC, it is clear that advective heat convergence is a key component. This has been shown by both observation- and model-based studies (Doney et al. 2007; Grist et al. 2010; Buckley et al. 2014, 2015; Piecuch and Ponte 2012; Piecuch et al. 2017; Roberts et al. 2017; Small et al. 2020).

A series of studies have shown that the balance between atmospheric forcing and forcing by ocean dynamics depends on the spatial resolution at which the budget is determined (Kirtman et al. 2012; Bishop et al. 2017; Small et al. 2019, 2020). By using spatial smoothing, Bishop 
et al. (2017) show that the importance of ocean-driven variability decreases with increasing spatial scale. This suggests that ocean-driven variability is mainly represented by small-scale features such as eddies. The spatial dependence was further confirmed in climate models for the relationship between SST and surface heat fluxes (Small et al. 2019) and for the upper ocean heat budget (Small et al. 2020). Similarly, there is a dependence on the temporal scale. While for monthly to seasonal anomalies atmospheric forcing is the dominant term, ocean dynamics becomes more important in establishing interannual and decadal variations in SST and upper OHC (Buckley et al. 2014, 2015). The time scale at which a switch occurs from an atmospheric- to an oceanic-driven scenario is regionally dependent (Buckley et al. 2015). By using a low-pass filter Bishop et al. (2017) show that importance of ocean-driven variability increases with increasing time scale. Small et al. (2019) expands the time-dependency to sub-monthly variability and show that the ocean-driven signal becomes relevant in the WBCs for time scales longer than 5 days.

The sensitivity to temporal and/or spatial scale has been either focused on particular ocean regions, such as the North Atlantic (Buckley et al. 2014, 2015), or on the global scale for the sea surface using observation-based analyses (Bishop et al. 2017; Small et al. 2019) and subsurface OHC variability based on climate models (Small et al. 2020). In this paper, we use an ocean model that assimilates ocean observations and examine the global distribution of regression coefficients for key drivers of ocean temperature variation. As a key additional step, we allow the data to tell us which regions share common dynamics via a clustering approach.

Our paper is organized as follows. Section 2 describes the ocean state estimate and the diagnostics used to describe heat content variability. An anomaly heat budget equation is then derived which is used to describe the temperature tendency anomaly as the sum of distinct variations in ocean heat processes (i.e., forcing, advection and diffusion). In Section 3, we present a local heat budget analysis for the upper ocean as defined by the wintertime MLD. The focus here was on evaluating 
the relative importance of each budget term as a driver of changes in OHC. With this analysis we introduce the covariance ratio, which quantifies the contribution of each budget term to the total variability of temperature. We show that the advection term is the most important driver of heat content in the tropics, while at higher latitudes forcing is increasingly relevant. We also performed a linearization of the advection terms that showed anomalous advection of the mean temperature field to be the main driver of temperature variability for the ocean in general. Section 4 presents heat budget variation at different spatial and temporal scales in order to evaluate the contribution of each budget term to the total budget at a range of vertical (i.e., depth) scales and horizontal and temporal (i.e., monthly to decadal) resolutions. We show a shift in the balance of heat budget terms with temporal scale, with forcing being relevant to $\mathrm{OHC}$ variability at short time scales but decreasing in relevance at longer time scales where advection becomes more important. Similarly, the analysis reveals that the balance of terms in the original $1^{\circ}$ grid shifts with increasing spatial aggregation, although the relative importance of each term to the overall budget does not change within a given zonal band. In Section 5 we introduce an unsupervised machine-learning approach to defining ocean regions based on coherent patterns in the local heat budget. The study's findings are further discussed in Section 6, with concluding remarks and suggestions for future work.

\section{ECCOv4 ocean state estimate and heat budget diagnostics}

In this paper, we conduct an investigation of the drivers of variability in $\mathrm{OHC}$ using the Estimating the Circulation and Climate of the Ocean (ECCO) consortium state estimate. The third release of version 4 (ECCOv4) was used, which provides a physically consistent ocean state estimate covering the period 1992-2015. Its solution is the output of the Massachusetts Institute of Technology general circulation model (MITgcm) assimilated to available observations for the period 1992 to 2015, which has been thoroughly assessed and found to be a coherent and accurate representation 
of the ocean state (Forget et al. 2015). In addition to providing closed tracer budgets, ECCOv4 offers detailed diagnostic information about the simulation, making it possible to identify the contributions of specific mechanisms to those budgets. Because of the model's conservation rules, there are no unidentified sources of heat, which makes ECCOv4 well suited as a reanalysis in order to investigate heat content variability in the ocean over recent decades.

The diagnostic outputs include monthly mean fields from January 1992 to December 2015 for all relevant terms to formulate the heat budget. In addition, diagnostics include monthly snapshots of temperature and sea surface height (taken at the beginning and end of each month). Both the mean and snapshot fields are presented in the Lat-Lon-Cap grid (i.e., LLC90) configuration, which is organized in 12 tiles with each tile including 90 by 90 grid cells (Forget et al. 2015). Horizontal grid spacing is irregular, with an average resolution of $1^{\circ} \times 1^{\circ}$. The grid size in LLC90 ranges from $40-50 \mathrm{~km}$ at polar to subpolar latitudes, to around $110 \mathrm{~km}$ towards the equator. Vertical spacing comprises 50 levels of thickness from $10 \mathrm{~m}$ at the surface to $456.5 \mathrm{~m}$ for the deepest layer.

\section{a. Anomaly heat budget in ECCOv4}

OHC variability is described here with the anomaly budget of temperature, whose terms are directly derived from diagnostic output of ECCOv4. The budget equation for temperature can be expressed in a general form as

$$
\frac{\partial \theta}{\partial t}+\nabla \cdot(\theta \mathbf{u})=-\nabla \cdot \mathbf{F}_{\text {diff }}+F_{\text {forc }}
$$

The temperature budget includes the change in temperature over time $\left(\frac{\partial \theta}{\partial t}\right)$, the convergence of heat advection $(-\nabla \cdot(\theta \mathbf{u}))$ and heat diffusion $\left(-\nabla \cdot \mathbf{F}_{\text {diff }}\right)$, plus downward heat flux from the atmosphere $\left(F_{\text {forc }}\right)$. Note that in our definition both latent and sensible air-sea heat fluxes, as well as longwave and shortwave radiation, is contained within $F_{\text {forc }}$. In order to derive the anomaly 
budget of temperature, first the budget equation of the monthly climatological mean temperature is determined, which can be done by recognizing that each variable can be expressed as a monthly mean plus its anomaly (i.e., climatology + seasonal anomaly). The monthly mean budget is derived by applying Reynolds averaging to Equation 1, and replacing each term by its monthly mean plus anomaly. The monthly mean and anomaly of variable $X$ are denoted as $\bar{X}^{m}$ and $X^{\prime}$, respectively. The monthly anomaly budget is then derived by subtracting the monthly mean equation from Equation 1, which removes the mean seasonal cycle and returns the month-to-month interannual variability. The central equation for the budget analysis is thus

$$
\frac{\partial \theta^{\prime}}{\partial t}=F_{\text {forc }}{ }^{\prime}-\nabla_{h} \cdot\left(\mathbf{u}^{\prime} \bar{\theta}^{m}\right)-\frac{\partial}{\partial z}\left(w^{\prime} \bar{\theta}^{m}\right)-\nabla_{h} \cdot\left(\overline{\mathbf{u}}^{m} \theta^{\prime}\right)-\frac{\partial}{\partial z}\left(\bar{w}^{m} \theta^{\prime}\right)-\nabla \cdot\left(\mathbf{u}^{\prime} \theta^{\prime}-{\overline{\mathbf{u}^{\prime} \theta^{\prime}}}^{m}\right)-\nabla \cdot \mathbf{F}_{\mathrm{diff}^{\prime}}+R
$$

The first term on the right-hand side of Equation $2\left(F_{\text {forc }}{ }^{\prime}\right)$ is anomalous forcing (i.e., anomalous air-sea heat flux). The convergence of the heat advection anomaly is described as the sum of terms resulting from the temporal decomposition of the advective fluxes. The advective heat flux is decomposed to a linear term due to temporal anomalies of the velocities, a linear term due to anomalies in temperatures, and a nonlinear term due to the covariance between the two anomalies. Furthermore, the two linear terms are separated into horizontal and vertical components. Technically, advective heat transport should only be calculated for flows with zero net mass transport (Warren 1999). However, we find it informative to separate horizontal and vertical components, recognizing that only the sum of these components has zero net mass transport. The analysis of these components is mainly provided as supplementary figures. Readers who disagree with this choice can simply disregard this part of analysis and focus on the sum of the two components. This 
detailed decomposition of the advective fluxes, beyond what was done by Small et al. (2020), is a novel aspect of our study.

The first two advective terms are horizontal $\left(-\nabla_{h} \cdot\left(\mathbf{u}^{\prime} \bar{\theta}^{m}\right)\right)$ and vertical $\left(-\frac{\partial}{\partial z}\left(w^{\prime} \bar{\theta}^{m}\right)\right)$ heat fluxes caused by velocity anomalies acting on the mean temperatures. The following two terms are horizontal $\left(-\nabla_{h} \cdot\left(\overline{\mathbf{u}}^{m} \theta^{\prime}\right)\right)$ and vertical $\left(-\frac{\partial}{\partial z}\left(\bar{w}^{m} \theta^{\prime}\right)\right)$ heat flux due to mean velocities acting on temperature anomalies. The nonlinear advective term $\left(-\nabla \cdot\left(\mathbf{u}^{\prime} \theta^{\prime}-{\overline{\mathbf{u}^{\prime} \theta^{\prime}}}^{m}\right)\right)$ describes the difference in advection given by the covariation between the velocity and temperature anomalies and the climatological mean of that covariation. Finally, Equation 2 includes the anomalous convergence of diffusion $\left(-\nabla \cdot \mathbf{F}_{\text {diff }}{ }^{\prime}\right)$ and a residual term $(R)$.

It should be noted that the derivation of this anomaly heat budget necessitates a residual term to yield an exact balance. The velocity terms in Equation 2 are the residual mean velocities containing both the resolved (Eulerian) and parameterized eddy induced transport. Because the advective temperature flux is derived with monthly-averaged model outputs of mass weighted velocities and temperature, the budget terms miss the effect of submonthly covariation. Furthermore, the derivation neglects temporal decomposition of the scaling factor corresponding to the non-linear free surface in ECCOv4 (Adcroft and Campin 2004; Campin et al. 2004). The residual term in Equation 2 therefore resolves these issues by accounting for any variability that is ignored in the offline estimation of the advective fluxes. The residual also includes the effects of numerical diffusion, which arise due to the model's advection scheme (Hill et al. 2012; Megann 2018). The flux due to effective numerical diffusion is present in the model's diagnostics of the full advective flux, but not in our linearized reconstruction of the flux. As shall be shown, the residual is small in virtually all instances. 


\section{Covariance analysis of local heat budget}

The ECCOv4 outputs permit calculation of the anomaly budget time series at each point in the global 3D grid. This yields too much information to comprehend or visualize, so to understand which terms drive heat content variability, we consider the correlation between the actual tendency, given by the left-hand side of (2) and denoted $y$, and each individual term on the right-hand side of the equation, denoted $x$. Similar forms of analysis were applied by Small et al. (2020), Small et al. (2019), and Doney et al. (2007).

We define the covariance ratio for a particular term $x$ as

$$
r_{x}=\frac{\sigma(x, y)}{\sigma(y)^{2}}
$$

where $\sigma(x, y)$ is the covariance between $x$ and $y$ and $\sigma(y)^{2}$ is the variance of $y$. In any particular heat budget, the covariance ratio describes the contribution of each budget term to the total temperature tendency. Since the total tendency is the sum of all the budget terms, the sum of the covariance ratios must equal one. This is true regardless of whether or not the terms of the budget are linearly independent (as in fact they are not); this decomposition is a physical, rather than statistical, analysis. A positive covariance ratio implies a positive contribution (and correlation) to the total tendency, and a negative value implies a negative contribution (and an inverse correlation) to the total tendency. Equation 3 is used to separately evaluate the relevance of each term (or sum of terms) in the anomaly heat budget (Equation 2) discretized into a selection of temporal scales and considering various horizontal and vertical scales.

$\mathrm{OHC}$ variability is first investigated for each grid point at the original temporal (monthly) resolution where the anomaly heat budget terms are integrated over the climatological winter mixed layer depth (hereafter referred as winter MLD). The winter MLD (Figure S1) defines a bottom boundary of the upper ocean that varies spatially but is fixed in time. This isolates the 
layer that is in exchange with the atmosphere on an annual time scale; this is the layer that is most relevant for and climate variability (Buckley et al. 2014, 2015). In this layer, we expect only minor influences on the heat budget by vertical mixing and entrainment.

The global distributions of the covariance ratios for the main terms (Figure 1) clearly shows that the balance in the anomaly heat budget is largely between anomalous forcing $\left(F_{\text {forc }}{ }^{\prime}\right)$ and advection $\left(-\nabla \cdot(\mathbf{u} \theta)^{\prime}\right)$. There are distinct global patterns of covariance ratios of the budget terms that are to the first order latitudinal. The covariance ratios for $F_{\text {forc }}{ }^{\prime}$ are essentially zero in the tropics but dominate regions at approximately $20^{\circ} \mathrm{N}$ and $20^{\circ} \mathrm{S}$ as well as in the Arctic and Antarctic (Figure 1a). In contrast, $-\nabla \cdot(\mathbf{u} \theta)^{\prime}$ reveals a broad pattern of high covariance ratios in the tropics and much lower covariance ratios in the subtropics and at polar and subpolar latitudes, though $-\nabla \cdot(\mathbf{u} \theta)^{\prime}$ is relevant for most of the extratropical ocean (Figure 1b).

Figures 1c-e are derived by the temporal decomposition of $-\nabla \cdot(\mathbf{u} \theta)^{\prime}$ into anomalous advection caused by anomalies in circulation $\left(-\nabla \cdot\left(\mathbf{u}^{\prime} \bar{\theta}^{m}\right)\right)$, temperature $\left(-\nabla\left(\overline{\mathbf{u}}^{m} \theta^{\prime}\right)\right)$, or covariation of anomalies in both $\left(-\nabla \cdot\left(\mathbf{u}^{\prime} \theta^{\prime}-{\overline{\mathbf{u}^{\prime} \theta^{\prime}}}^{m}\right)\right.$, referred hereafter as the nonlinear advective term). Variability in advective heat convergence is largely driven by $-\nabla \cdot\left(\mathbf{u}^{\prime} \bar{\theta}^{m}\right)$. In discrete locations associated with boundary, circumpolar and equatorial currents, $-\nabla\left(\overline{\mathbf{u}}^{m} \theta^{\prime}\right)$ is relevant (Figure 1d).

\section{Large compensation between horizontal and vertical components of $-\nabla \cdot\left(\mathbf{u}^{\prime} \bar{\theta}^{m}\right)$ while $-\nabla\left(\overline{\mathbf{u}}^{m} \theta^{\prime}\right)$} is mostly driven by the horizontal component (Figure 3, top row). The vertical component of the anomalous advection of mean temperature $\left(-\frac{\partial}{\partial z}\left(w^{\prime} \bar{\theta}^{m}\right)\right)$ dampens the effect of the horizontal component and generally contributes to a reduction in the total variability. As $-\nabla_{h} \cdot\left(\mathbf{u}^{\prime} \bar{\theta}^{m}\right)$ contributes to a positive or negative temperature anomaly, $-\frac{\partial}{\partial z}\left(w^{\prime} \bar{\theta}^{m}\right)$ counteracts this effect. This compensation is particular evident in the tropical ocean, where $-\nabla_{h} \cdot\left(\mathbf{u}^{\prime} \bar{\theta}^{m}\right)$ and $-\frac{\partial}{\partial z}\left(w^{\prime} \bar{\theta}^{m}\right)$ are exact opposite sign and same magnitude (Figure 3). In the extra tropics it is $-\nabla_{h} \cdot\left(\mathbf{u}^{\prime} \bar{\theta}^{m}\right)$ that determines the sign of the total advective convergence $(-\nabla \cdot(\mathbf{u} \theta))$, because the mostly positive 
covariance ratios for $-\nabla \cdot(\mathbf{u} \theta)^{\prime}$ are reflected by $-\nabla_{h} \cdot\left(\mathbf{u}^{\prime} \bar{\theta}^{m}\right)$, and the compensation by $-\frac{\partial}{\partial z}\left(w^{\prime} \bar{\theta}^{m}\right)$ is only a fraction of $-\nabla_{h} \cdot\left(\mathbf{u}^{\prime} \bar{\theta}^{m}\right)$.

The covariance ratio of the nonlinear advective term is effectively zero except for some variability in the tropical western Pacific and tropical eastern Indian Ocean (Figure 1e). These resemble the spatial pattern in winter MLD (Figure S1). The diffusion term $\left(-\nabla \cdot \mathbf{F}_{\text {diff }}{ }^{\prime}\right)$ exhibits only minor influence on the heat budget. Only in the polar latitudes there are some regions such as the Beaufort, Ross and Weddell Sea, with higher covariance ratios, and these are associated with very shallow winter MLD, thus, representing an exception to the assumptions of negligible vertical mixing above the winter MLD. Finally, the residual term is close to zero everywhere (Figure S2), confirming that our ocean heat budget can be essentially closed without accounting for submonthly covariation and approximation of the scaling factor.

In conclusion, the monthly anomaly heat budget integrated over the winter MLD on the original ECCOv4 grid is largely determined by anomalies in sea surface heat flux and anomalous advection of the mean, while advection of temperature anomalies play a role only in specific regions of relatively strong currents (e.g., western boundary currents). In Section 5, we seek further insight into the physics of these patterns by using cluster analysis to identify dynamically similar regions. First, however, we examine the scale sensitivity of these patterns.

\section{Dependence on spatial and temporal scale}

In this section, we explore the sensitivity of the covariance-ratio analysis to different choices regarding spatial and temporal aggregation. The point of this is to investigate whether the patterns identified in Section 3 and corresponding conclusions about the heat budget are robust over space and time scales, or whether qualitative changes emerge as we consider different scales. 


\section{a. Depth of integration}

In contrast to integrating over the winter MLD, we also investigated the balance between budget terms integrated over fixed depths. The aim of this is to understand how the heat budget varies as one considers deeper and deeper portions of the ocean. We know, for example, that all vertical fluxes must eventually vanish as we approach the bottom, but how deep must we go to see this? Small et al. (2019) and Small et al. (2020) focused only on the upper ocean in their analysis, leaving this question open.

The covariance ratios for each term in the heat budget were calculated for a range of depths (i.e., $50 \mathrm{~m}, 100 \mathrm{~m}, 300 \mathrm{~m}, 700 \mathrm{~m}, 2000 \mathrm{~m}$, and $6000 \mathrm{~m} / \mathrm{full}-\mathrm{depth})$ in order to describe the change in the relative importance of different mechanisms as vertical integration is varied. The principal drivers of the heat budget are consistently $F_{\text {forc }}{ }^{\prime}$ and $-\nabla \cdot(\mathbf{u} \theta)^{\prime}$, but the balance between these mechanisms changes substantially according to the specific depth scale (Figure 2 ). As expected, $F_{\text {forc }}{ }^{\prime}$ dominates the heat budget at shallower depths of integration (i.e., $50 \mathrm{~m}, 100 \mathrm{~m}$ ) in almost every region, with a shift at increasing depth from $F_{\text {forc }}{ }^{\prime}$ to $-\nabla \cdot(\mathbf{u} \theta)^{\prime}$ as the dominant factor. Overall the most striking shift in patterns is from 100 to $300 \mathrm{~m}$, while the change in patterns is more subtle when shifting the integration depths from the upper $300 \mathrm{~m}$ to deeper layers. This is clearly related to the spatial relationship between depth of integration and extent of vertical mixing.

At shallow depths (i.e., 50 and $100 \mathrm{~m}$ ) the pattern of covariance ratios for all budget terms closely resembles the covariance pattern of the winter MLD (Figure 1) in the lower latitudes. In the higher latitudes, the covariance patterns for deeper layers (i.e., $>300 \mathrm{~m}$ ) in Figure 2 resemble those in Figure 1. This is mostly due to the spatial pattern of the winter MLD, which to the first order is deeper in the high latitudes (i.e., 200 to $1000 \mathrm{~m}$ ) and shallower in the low latitudes (i.e., $<200 \mathrm{~m}$ ). 
When integrated at $300 \mathrm{~m}$ and greater depths, $-\nabla \cdot\left(\mathbf{u}^{\prime} \bar{\theta}^{m}\right)$ dominates in all regions outside of the high latitudes. The shift to the increasing significance of $-\nabla \cdot(\mathbf{u} \theta)^{\prime}$ with depth is mostly due to the anomalous circulation term, while the patterns associated with mean circulation of anomalies is relatively insensitive to the depth of integration. The only exception is the relevance of $-\nabla\left(\overline{\mathbf{u}}^{m} \theta^{\prime}\right)$ in the tropics seen in Figure 1 and for the upper 50-100 m in Figure 2, which disappear when integrating over deeper layers. This is consistent with very shallow depth scale of the equatorial mean jets.

The effect of $-\nabla \cdot \mathbf{F}_{\text {diff }}$ is only noticeable for the upper $50 \mathrm{~m}$, which is evident when the fixed depth integration occurs within the winter MLD and is indicative of $-\nabla \cdot \mathbf{F}_{\text {diff }}{ }^{\prime}$ being important only in areas of deep winter convection (i.e., at high latitudes and especially in regions with deep convective sites such as the SPNA, Nordic Seas and Southern Ocean).

If Figure 3, we decompose advection into horizontal and vertical components. The compensation (i.e., anticorrelation) between the horizontal and vertical components of advection are particularly prominent at $50 \mathrm{~m}$ and $100 \mathrm{~m}$ in the lower latitudes (Figure 3). Again, there is a stark pattern shift when moving from $100-300 \mathrm{~m}$ to $700 \mathrm{~m}$, at which point there is much less compensation in the lower latitudes and more pronounced compensation in the mid-latitudes such as in the subtropical gyres. Integrating over deeper layers (i.e., 2000 to $6000 \mathrm{~m}$ ) leads to vanishingly small vertical convergences.

It is interesting to note that the anticorrelation between horizontal and vertical components only applies to anomalous circulation $\left(\mathbf{u}^{\prime} \bar{\theta}^{m}\right)$, but not to advection of temperature anomalies by the mean flow $\left(\overline{\mathbf{u}}^{m} \theta^{\prime}\right)$. This suggests a mechanism underlying this compensation: volume conservation. The continuity equation for the anomalous flow, $\nabla_{h} \cdot \mathbf{u}^{\prime}+\frac{\partial w^{\prime}}{\partial z}=0$, states that convergence of horizontal transport and vertical transport must be anticorrelated. The anticorrelation evidently carries over to the convergence of heat fluxes as well. 


\section{b. Temporal scale}

The ocean heat anomaly budget up to this point was only evaluated at monthly resolution. Considering the upper ocean $(<300 \mathrm{~m})$ and at higher latitudes, $F_{\text {forc }}{ }^{\prime}$ is the major term in determining total tendency at this relatively short time scale. Previous studies have shown that only at longer time scales do certain mechanisms, such as geostrophic or diffusive heat transport, become relevant (Buckley et al. 2014). Similarly, Bishop et al. (2017) showed that SST variability becomes increasingly driven by ocean processes. Therefore a shift in the balance of terms within the heat budget is expected as temporal scale increases. To assess any changes in the balance of terms at longer temporal scales, the budget was determined by temporally aggregating time series at 3-month, 6-month, 1-year (i.e., annual), 2-year, 3-year, 4-year, 5-year (i.e., pentadal) and 10-year (i.e., decadal) intervals. The supplementary materials provide an illustration of the change in the time series of heat budget terms according to temporal aggregation scale for the subpolar North Atlantic (Figure S4). The aim of these multiple temporal aggregations was to clearly illustrate shifts in the balance of budget terms and whether these occur gradually or appear as a sudden shift at a particular timescale. Rather than focusing on a particular ocean region, we focused instead on describing globally how the budget terms shift with temporal scale across different latitude bands and for different depths of integration. One caveat of this approach is that, as we aggregate to coarser temporal scales, the timeseries have fewer and fewer points, and the correlations become more noisy.

Covariance ratios were averaged into $10^{\circ}$ latitude bins to derive zonal means (Figure 4). These confirm that the balance of the heat budget is dominated by $-\nabla \cdot\left(\mathbf{u}^{\prime} \bar{\theta}^{m}\right)$ and $F_{\text {forc }}{ }^{\prime}$. With longer time scales, the relevance of $-\nabla \cdot\left(\mathbf{u}^{\prime} \bar{\theta}^{m}\right)$ increases. For annual and pentad averages, $-\nabla \cdot\left(\overline{\mathbf{u}}^{m} \theta^{\prime}\right)$ also becomes more important, especially in the southern high latitudes (corresponding to the 
Southern Ocean and ACC). Over the winter MLD (top row in Figure 4), the covariance ratios of combined advection terms are only dominant near the equator between $10^{\circ} \mathrm{S}$ to $10^{\circ} \mathrm{N}$. The combined covariance ratios are around 0.5 in the mid latitudes, and show only minor influence across the higher latitude bands. $F_{\text {forc }}{ }^{\prime}$ remains dominant in the high northern latitudes $\left(>60^{\circ} \mathrm{N}\right)$ in most cases even as its relevance tends to decline with longer time scales. $-\nabla \cdot \mathbf{F}_{\text {diff }}{ }^{\prime}$ becomes increasingly important in the high latitudes at longer time scales. In the northern high latitudes it presents a dampening effect (i.e., it has a negative covariance ratio), while in the southern high latitudes $\left(>60^{\circ} \mathrm{S}\right)$ it increasingly determines the total tendency. This is consistent with the spatial distributions presented in Figure 1f, where the influence of $-\nabla \cdot \mathbf{F}_{\text {diff }}{ }^{\prime}$ is evident in the marginal seas of Antarctica, the western SPNA (Labrador Sea) and the Nordic Seas, and shows compensation in specific parts of the Arctic Ocean.

We next addressed the question, how does integration of the heat budget over different depth levels (i.e., the winter MLD vs. the upper 300 or $700 \mathrm{~m}$ ) affect how the budget term balance changes with different time scales? There are clear changes in covariance ratio patterns when moving from integration over the wintertime MLD to a fixed depth of integration. When integrating at $300 \mathrm{~m}$ or $700 \mathrm{~m}$, the influence of $-\nabla \cdot\left(\mathbf{u}^{\prime} \bar{\theta}^{m}\right)$ increases at all latitudes, with the zonal mean covariance ratios being highest in the lower latitudes $\left(30^{\circ} \mathrm{S}\right.$ to $\left.30^{\circ} \mathrm{N}\right)$. However, the overall shift towards the budget being more driven by $-\nabla \cdot(\mathbf{u} \theta)^{\prime}$ over longer time scales is consistent both in the case of integrating over the winter MLD and over a fixed depth. The picture is remarkably similar between $300 \mathrm{~m}$ and $700 \mathrm{~m}$, and also at deeper depths $(2000 \mathrm{~m})$ or full depth (omitted in Figure 4 because they are very similar to the 300 and 700 m patterns). As seen in Figure 2 the zonal band where OHC variability is mainly advection-driven expands to higher latitudes with increasing depth of integration.

When integrating over $300 \mathrm{~m}$ or deeper, there is no apparent compensation (cancelling positive and negative terms) except for the pentad averages. There are multiple terms whose zonal mean of 
covariance ratios are negative, occurring at latitude $30^{\circ} \mathrm{S}$ to $60^{\circ} \mathrm{S}$ (corresponding to the Southern Ocean) and at $70^{\circ} \mathrm{N}$ (corresponding to the Nordic Seas). This indicates that in these latitudes there can be strong anticorrelation at pentad time scale for terms that usually contribute to the total tendency (i.e., have positive covariance ratios). At latitude $70^{\circ} \mathrm{N}$, the nonlinear advective term shows a strong compensation which is not apparent at higher frequencies (monthly and annual). At $60^{\circ} \mathrm{S}$ we see that $-\nabla \cdot\left(\mathbf{u}^{\prime} \theta^{m}\right)$, which is generally contributing to total tendency, dampens variability by counteracting $-\nabla\left(\overline{\mathbf{u}}^{m} \theta^{\prime}\right)$ and $F_{\text {forc }}{ }^{\prime}$.

\section{c. Horizontal scale}

The balance of contributing terms in the heat budget equation varies according to the spatial and temporal scales on which the terms are derived. The remaining question is how the importance of each budget term changes as spatial aggregation changes from the original $1^{\circ} \times 1^{\circ}$ grid to increasingly coarse aggregation scales (e.g., $\left.2^{\circ} \times 2^{\circ}, 5^{\circ} \times 5^{\circ}, 10^{\circ} \times 10^{\circ}\right)$. The dependence on horizontal scale has been pointed out by previous studies focusing on the surface ocean (Bishop et al. 2017; Small et al. 2019) and in climate models (Small et al. 2020), which showed that ocean transport is more relevant for higher resolutions. Table 1 lists the global average of covariance ratios of each budget term listed for each spatial aggregation scale, starting with the original resolution $(1 \times 1)$ to a maximum binning level of $90 \times 90$. In general, global mean covariance ratios for the upper ocean are sensitive to spatial scale, changing gradually when spatially aggregating the fields (Table 1).

There is a notable increase in $F_{\text {forc }}{ }^{\prime}$ with larger aggregation scales, accompanied by a concomitant decrease in the contribution by $-\nabla \cdot(\mathbf{u} \theta)^{\prime}$. The relevance of both linear advective terms, $-\nabla \cdot\left(\mathbf{u}^{\prime} \bar{\theta}^{m}\right)$ and $-\nabla\left(\overline{\mathbf{u}}^{m} \theta^{\prime}\right)$, declines as the aggregation scale increased. The shift in the balance of terms ceases after $15 \times 15$ and remains relatively constant up to the $90 \times 90$ level of aggregation, the upper limit of coarsening for this exercise. The greatest contribution by $F_{\text {forc }}$ (on a global average basis) is at 
$30 \times 30$, where it represents around $3 / 4(76 \%)$ of the total, while $-\nabla \cdot(\mathbf{u} \theta)^{\prime}$ is around a $1 / 4(25 \%)$.

The global mean covariance ratios for $-\nabla \cdot \mathbf{F}_{\text {diff }}{ }^{\prime}$, the nonlinear advection term, and the residual remain effectively zero across all spatial scales. A trend for increasing relevance of $-\nabla \cdot \mathbf{F}_{\text {diff }}{ }^{\prime}$ at larger aggregation scales is evident, but remains minor when covariance ratios are averaged over the whole globe.

Similar to Figure 4, the covariance ratios are again averaged in $10^{\circ}$ latitude bins and are plotted against latitude to illustrate the zonal balance between $F_{\text {forc }}{ }^{\prime}$ and $-\nabla \cdot(\mathbf{u} \theta)^{\prime}$ with changing horizontal resolution, from the original $1^{\circ} \times 1^{\circ}$ resolution to $30 \times 30$ aggregation (Figure 5). $F_{\text {forc }}{ }^{\prime}$ and $-\nabla \cdot(\mathbf{u} \theta)^{\prime}$ were determined over the winter MLD and the upper $300 \mathrm{~m}$ and $700 \mathrm{~m}$ depth. Note that the coarsest resolution is set here at $30 \times 30$, because any coarser resolution fails to retain the latitudinal pattern. As well, there is little change in the global balance of terms beyond that horizontal aggregation scale (Table 1). The zonal means of covariance ratios show similar sensitivity across all latitudes with only a few exceptions. $F_{\text {forc }}{ }^{\prime}$ shifts slightly more in the high latitudes (especially in the Northern Hemisphere). The strongest shifts in the covariance ratios for $-\nabla \cdot(\mathbf{u} \theta)^{\prime}$ are in the mid-latitudes, especially in the Southern Hemisphere. $-\nabla \cdot(\mathbf{u} \theta)^{\prime}$ remains the main contributor in the low latitudes even at the largest aggregation scales.

Although a clear shift in the covariance ratios is evident, the overall balance across latitude remains the same. Where forcing is dominant (as in the high to mid latitudes) at the native grid resolution $(1 \times 1)$, it is still relevant at the coarsest resolutions $(30 \times 30)$. This remains true when looking at different temporal scales (i.e., monthly, annual or pentad averages) as well for different depths of integration (i.e., winter MLD, $300 \mathrm{~m}, 700 \mathrm{~m}$ ). While the individual terms may shift, there are only a few cases where spatial aggregation causes a change in the overall balance of terms. For the winter MLD (top row), pentad scale includes large compensation by $-\nabla \cdot \mathbf{F}_{\text {diff }}^{\prime}$ at $70^{\circ} \mathrm{N}$, which is associated with both $F_{\text {forc }}{ }^{\prime}$ and $-\nabla \cdot(\mathbf{u} \theta)^{\prime}$ having covariance much greater than 1.0. Whereas in 
the upper $300 \mathrm{~m}$ and $700 \mathrm{~m}$, pentad averages of $F_{\text {forc }}{ }^{\prime}$ at $70^{\circ} \mathrm{N}$ result in high covariance ratios $(>1.0)$ only at smaller spatial scales. At these fixed depths, $-\nabla \cdot(\mathbf{u} \theta)^{\prime}$ is affected by spatial aggregation as covariance ratios shift from positive to negative values (Figure 5).

As the zonal means of covariance ratios in Figure 4 suggest, the contribution of $-\nabla \cdot(\mathbf{u} \theta)^{\prime}$ (in particular $\left.-\nabla\left(\overline{\mathbf{u}}^{m} \theta^{\prime}\right)\right)$ increases as the temporal scale increases. The same can be observed in Figure 5, in which the latitude band where the zonal mean covariance ratio of $-\nabla \cdot(\mathbf{u} \theta)^{\prime}$ is greater than $F_{\text {forc }}{ }^{\prime}$ expands as the temporal scale increases from monthly to pentad averages. This is unambiguous at 300 and $700 \mathrm{~m}$, while it is less obvious but discernible for the winter MLD. This has important implications for the interpretation of longer timescale (e.g., decadal trends) in OHC. As these findings suggest, $-\nabla \cdot(\mathbf{u} \theta)^{\prime}$ should play a more important role when focusing on decadal trends of heat content (Lee et al. 2011; Yeager et al. 2012; Zhang 2017).

The varying balance of the budget terms at different integration depths and aggregation scales raise the question of at what spatial scale $F_{\text {forc }}{ }^{\prime}$ becomes the dominant term. $F_{\text {forc }}{ }^{\prime}$ is dominant within the winter MLD, but just by integrating over the upper $300 \mathrm{~m},-\nabla \cdot(\mathbf{u} \theta)^{\prime}$ becomes dominant outside the high latitudes. As we see in Figure 5, for upper $300 \mathrm{~m}$ (and deeper depths) the contribution of $-\nabla \cdot(\mathbf{u} \theta)^{\prime}$ remains distinctly larger than $F_{\text {forc }}{ }^{\prime}$ at most low to mid latitude basins at wide spatial aggregation scales. It must be that for the highest level of aggregation (i.e., summing the budget terms over the global scale), the contribution of $-\nabla \cdot(\mathbf{u} \theta)^{\prime}$ (and $-\nabla \cdot \mathbf{F}_{\text {diff }}{ }^{\prime}$ ) to the heat budget must go to zero. Thus, as the aggregation scale increases, the balance of terms should shift such that the $F_{\text {forc }}{ }^{\prime}$ term increases in relative importance (with $-\nabla \cdot(\mathbf{u} \theta)^{\prime}$ and $-\nabla \cdot \mathbf{F}_{\text {diff }}{ }^{\prime}$ increasingly less important). Yet as evident in Table 1 and Figure 5, contribution of $-\nabla \cdot(\mathbf{u} \theta)^{\prime}$ is still relevant at very coarse resolutions (corresponding to roughly $90^{\circ} \times 90^{\circ}$ ) and is major at low to mid latitudes when integrating over a fixed depth of $>300 \mathrm{~m}$. 
The heat budget was also evaluated for three ocean basins (i.e., Pacific, Atlantic, Indian) as a representation of highest spatial aggregation besides the global integral. The spatial masks we use for the ocean basin are provided by the gcmfaces toolbox Forget et al. (2015) and are shown in Figure S5. The largest contribution to the basin-scale heat budget over the winter MLD is clearly by $F_{\text {forc }}{ }^{\prime}$, but $-\nabla \cdot(\mathbf{u} \theta)^{\prime}$ is also relevant (Table S1). Interestingly, for the Pacific and Atlantic basins, it is mainly the vertical advection, specifically $-\frac{\partial}{\partial z}\left(w^{\prime} \bar{\theta}^{m}\right)$ that is dominating the contribution by $-\nabla \cdot(\mathbf{u} \theta)^{\prime}$. This is consistent with the analysis of vertical heat transport by Liang et al. (2015).

The basin-wide heat budgets are further analysed for different depths and temporal scales for the main terms (Figure S6). Covariance ratios for $F_{\text {forc }}{ }^{\prime}$ are very close to 1.0 for the deep basins. The influence of $-\nabla \cdot(\mathbf{u} \theta)^{\prime}$ does not increase for greater integration depths, but it does become more important at longer time scales, especially in Atlantic and Indian basins. Across the three basins a clear shift occurs at $>2 \mathrm{~A}$ (Pacific), $>3 \mathrm{~A}$ (Atlantic) $>2-3 \mathrm{~A}$ (Indian). The shift in relevance is due to both greater relevance in $-\nabla \cdot\left(\mathbf{u}^{\prime} \bar{\theta}^{m}\right)$ and $-\nabla\left(\overline{\mathbf{u}}^{m} \theta^{\prime}\right)$ (Figure S7). Yet again, as shown in the local heat budget maps (Figures 1,2) and zonal means (Figure 4), most advective-driven variance is accounted by variability in $-\nabla \cdot\left(\mathbf{u}^{\prime} \bar{\theta}^{m}\right)$. The vertical components are considerable only at depths of integration $<300 \mathrm{~m}$ (Figure S8). Thus, the horizontal advection terms $\left(-\nabla_{h} \cdot\left(\mathbf{u}^{\prime} \theta^{m}\right)\right.$ and $\left.-\nabla_{h} \cdot\left(\overline{\mathbf{u}}^{m} \theta^{\prime}\right)\right)$ are important to consider for deep basin-wide ocean heat budgets on longer time scales.

\section{Classification of dynamical regimes}

The balance of terms in the upper ocean heat budget shows clear spatial patterns (Figure 1) which suggest distinct dynamical regimes, each associated with particular underlying mechanisms controlling heat content variability. Effectively summarizing dynamical regimes relevant to the ocean heat budget on a global scale is challenging given the overwhelming detail necessary to 
adequately describe each ocean region. Rather than splitting regions based on geographical features, we pursued an unsupervised machine learning technique to assess the global spatial pattern of $\mathrm{OHC}$ variability.

The k-means clustering algorithm is an efficient tool to reduce the spatial complexity of large datasets (Hartigan and Wong 1979; Gong and Richman 1995; Lund and Li 2009). A common application of clustering analysis in oceanography is in the identification of ecological provinces, which has been done in specific regions, such as the northwest Atlantic (Devred et al. 2007) and Southern Ocean (Ardyna et al. 2017), and globally (Sonnewald et al. 2020). A similar approach was used in a recent study in which the mean balance in the barotropic vorticity budget was analyzed (Sonnewald et al. 2019); however, that study focused on classifying the time-mean budgets. Our application of clustering is novel because it is applied to the covariance ratios, rather than the mean budget.

We applied k-means clustering to the covariance ratios of the three main heat budget terms $F_{\text {forc }}{ }^{\prime}$, $-\nabla \cdot(\theta \mathbf{u})$ and $-\nabla \cdot \mathbf{F}_{\text {diff }}{ }^{\prime}$, which were integrated over the winter MLD. The corresponding spatial patterns of the covariance ratios of the three budget terms are shown in Figure 1a,b and $f$. The dimensions of the parameter space (i.e., features) are defined by the covariance ratios of the three heat budget terms. The optimal number of clusters $(\mathrm{k}=5)$ was shown to minimize variation within each cluster and any increase in $\mathrm{k}$ did not yield further (significant) reduction. Each ocean grid point was assigned to a given cluster based on the proximity to the clusters's centroids within the parameter space.

The spatial distribution of the five global clusters is shown in Figure 6. Having divided the global ocean into these dynamical regions provides the opportunity for a physical interpretation of the drivers of heat content variability (Figure 7). $-\nabla \cdot(\mathbf{u} \theta)^{\prime}$ clearly dominates the heat budget in regions associated with cluster A. This is mainly because of the presence of strong currents near 
the equator, the ACC and western boundary currents. In the case of boundary currents and ACC also correspond to strong spatial gradients in temperature (Bishop et al. 2017).

Cluster B is dominated solely by $F_{\text {forc }}{ }^{\prime}$ and corresponds to regions where the winter MLD is relatively shallow (100-150 m, Figure S1). These are also regions where ocean velocities are generally weak and there are no strong spatial gradients in temperature. Due to the weak velocity fields there is no significant horizontal exchange within the mixed layer, and the heat content variability is driven by the atmosphere. Meanwhile cluster $\mathrm{C}$ represents the greatest area of the global ocean and a dynamical regime somewhere between clusters $\mathrm{A}$ and B in which both $F_{\text {forc }}{ }^{\prime}$ and $-\nabla \cdot(\mathbf{u} \theta)^{\prime}$ have major roles. Clusters $\mathrm{D}$ and $\mathrm{E}$ represent regions where diffusion is relevant, as in the Beaufort Gyre in the Arctic and the Antarctic marginal seas (i.e., the Ross and Weddell Seas). These regions are characterized by a very shallow winter MLD $(<100 \mathrm{~m}$, Figure S1) and can be considered outliers. Dynamical regimes associated with clusters A, B and C represent the vast majority of the ocean.

We further divided the clusters into basin-specific dynamical regimes (Figure S9) in order to investigate the heat budget on a regional basis. Particular regions can serve as examples of the key dynamical regimes. In the advection-dominated regions (i.e., cluster A) we identify the Kuroshio current and extension in the North Pacific (Figure S9, A1) and the Gulf Stream in the North Atlantic (Figure S9, A2). Here the heat budget will be dominated by the western boundary current. The other advection-driven regions are the tropical Indian (A3), Pacific (A4) and Atlantic (A5) as well as the ACC (A6). The selected forcing-dominated regions (i.e., cluster B) are in the subtropical Atlantic and Pacific (Figure S9, B1-B4). The representative regions for cluster C, where both $F_{\text {forc }}$ and $-\nabla \cdot(\mathbf{u} \theta)^{\prime}$ are relevant, were chosen from the North Atlantic, North and South Pacific and Indian basins (Figure S9, C1-C4). Budget analyses for the Arctic Ocean and Antarctic marginal seas (clusters D and E, respectively), where diffusion makes a substantial contribution, are also 
included. An exhaustive intercomparison of all of these regions is presented in the supplemental material for the interested reader (Table S3, Figures S10-S24), but the remaining part of this section will focus on a subset of representative regions.

When the heat budget for the Kuroshio current is calculated over the winter MLD, $F_{\text {forc }}{ }^{\prime}$ and $-\nabla \cdot(\mathbf{u} \theta)^{\prime}$ each contribute half of the variability (Table S3). Integrating below the winter MLD, $-\nabla \cdot(\mathbf{u} \theta)^{\prime}$ increasingly dominates the variability in the heat budget with increasing depth of integration, and there is a clear shift evident from 100 to $300 \mathrm{~m}$ (Figure 8). The shift towards $-\nabla \cdot(\mathbf{u} \theta)^{\prime}$ is especially notable at longer time scales, at which point it is the main driver with $F_{\text {forc }}{ }^{\prime}$ now counteracting $-\nabla \cdot(\mathbf{u} \theta)^{\prime}$ (i.e., $F_{\text {forc }}{ }^{\prime}$ has negative covariance ratios). At longer time scales, there is a clear anti-correlation between the variability due to velocity anomalies and variability due to temperature anomalies in the Kuroshio current (Figure 9). This is consistent with Buckley et al. (2015), who concluded that in regions where geostrophic currents are important, the decomposition between temperature and velocity variability is not meaningful.

As for the Kuroshio current and extension (region A1), the tropical Pacific (region A4) is advection-driven, with the distinction that $-\nabla \cdot(\mathbf{u} \theta)^{\prime}$ is much less sensitive to depth of integration and time scale (Figure 8) and there is no anticorrelation apparent between $-\nabla \cdot\left(\mathbf{u}^{\prime} \bar{\theta}^{m}\right)$ and $-\nabla\left(\overline{\mathbf{u}}^{m} \theta^{\prime}\right)$ (Figure 9). In the subtropical North Pacific, there is an abrupt shift from a forcing-dominated to an advection-dominated budget when moving from 100 to $300 \mathrm{~m}$ (which corresponds to crossing the winter MLD). This illustrates that even when $F_{\text {forc }}{ }^{\prime}$ is the dominant term within the winter MLD, integrating over deeper depths causes the budget to become advection-dominated (especially at longer time scales). This is the case for all B-regime regions (Figure S12). Similar to other dynamical regimes, the shift towards a more advection driven budget with longer time scales is apparent where at timescales $>2 \mathrm{~A},-\nabla \cdot(\mathbf{u} \theta)^{\prime}$ make up roughly half of the budget (Figure 9 and S12). In the northeast Pacific, the shift in budget terms with depth is more gradual (Figure 8). Here 
the winter MLD is at approximately $150 \mathrm{~m}$, so forcing remains significant at deeper integration depths, but only at shorter time scales (i.e., $>2 \mathrm{~A}$ ). At longer time scales there is a clear shift to advection-dominance.

The above comparison of regional budgets for different dynamical regimes shows that the balance of terms in each case is sensitive to the spatial (in this case, depth of integration) and temporal scale. This sensitivity is different for each region, but in most cases there is a clear decrease in the significance of $F_{\text {forc }}$ along with an increase in $-\nabla \cdot(\mathbf{u} \theta)^{\prime}$ at longer time scales. One exception is the tropical ocean regions where heat content variability is mostly driven by $-\nabla \cdot(\mathbf{u} \theta)^{\prime}$ across all time scales and depth levels. For all regions, diffusion is only relevant within the winter MLD. Although unique to the tropical regions, diffusion is mostly irrelevant even at shallow depths (Figure 8 and Figure S11).

\section{Conclusion}

This study investigated the contribution of individual mechanisms to $\mathrm{OHC}$ variability at a range of spatial and temporal scales. By employing ECCOv4, which is constrained by observations in a physically consistent way, the variability investigated in our analysis closely reflects the variability in the observed state of the ocean such as is described by ocean remote sensing and global observational networks (e.g., Argo). The work presented here includes novel approaches in which covariance ratios have been evaluated for a fully closed budget and have been used to define dynamical regimes. These approaches complement previous work describing factors influencing the ocean heat budget.

We have shown here that the balance in the upper ocean heat budget is mainly between anomalous surface forcing $\left(F_{\text {forc }}{ }^{\prime}\right)$ and convergence in anomalous advection $\left(-\nabla \cdot(\mathbf{u} \theta)^{\prime}\right)$. Furthermore, the temporal decomposition of mean versus anomalous heat advection provided new insights in the 
$\mathrm{OHC}$ variability. In particular, the decomposition allowed us to see that most of the OHC variability is due to anomalies in circulation $\left(-\nabla \cdot\left(\mathbf{u}^{\prime} \bar{\theta}^{m}\right)\right)$, while anomalies in the temperature field $\left(-\nabla\left(\overline{\mathbf{u}}^{m} \theta^{\prime}\right)\right)$ are only relevant to total heat content variability associated with specific regional features (e.g., boundary currents, circumpolar currents and equatorial currents). We also show that the diffusion and non-linear terms of the budget are for the most part negligible.

By using the covariance ratio of the main budget terms as the set of features in the clustering algorithm, we defined dynamical regimes such that each would feature similar underlying mechanisms controlling variability in anomalous heat fluxes. Instead of using the mean budget, we focused on variability in the seasonal anomalies to define regions that are in essence dynamically similar. Ocean regions where $-\nabla \cdot(\mathbf{u} \theta)^{\prime}$ dominates the heat budget generally have strong currents and mostly show strong gradients in SST (Bishop et al. 2017). We identified specific areas in the ocean where $F_{\text {forc }}{ }^{\prime}$ is the sole driver of the upper ocean heat budget. These correspond to regions where the winter MLD is relatively shallow and ocean currents are weak, and therefore resemble one dimensional surface forced layers of the ocean that don't have a lot of significant exchange with neighboring regions. The vast majority of the ocean, however, corresponds to regions with significant contribution by both $F_{\text {forc }}{ }^{\prime}$ and $-\nabla \cdot(\mathbf{u} \theta)^{\prime}$.

Advective convergence due to circulation anomalies is by far the dominant driver of ocean heat change in the tropics, while $F_{\text {forc }}{ }^{\prime}$ contributes to local heat content variability only at higher latitudes. Our observation of the overall global pattern of covariance ratios, where $F_{\text {forc }}{ }^{\prime}$ relevance is close to zero in the tropics, is consistent with Bishop et al. (2017), who showed a weak correlation at zero lag between SST tendency and surface heat flux in the tropics (i.e., surface heat flux has little effect on the tendency). Considering that their lead-lag correlations were not normalized to the overall magntidue of variability, the global patterns agree with the one presented in the more recent global assessment by Small et al. (2019) for the sea surface as well as the upper ocean Small 
et al. (2020). The fact that we have found these same relationships in a data-assimilating state estimate highlights the robustness of these mechanisms, and of the method itself.

By distinguishing between horizontal and vertical components of advection, we have shown that vertical advective flux largely compensates for the horizontal component of the local heat budget. This is observed in the spatial distribution of covariance ratio where the horizontal term is greater than 1.0 while for the vertical term it is negative (Figure 3). This simply indicates that convergence in the horizontal transport is correlated with divergence in the vertical transport (i.e., volume is conserved). Almost everywhere it is the horizontal component that is proportional to, and thus contribute to, the temperature tendency. Many studies have employed advection estimates from satellite data under the assumption that the horizontal component is sufficient to reconstruct ocean advection, but here we have taken the opportunity to directly test these assumptions by looking at the contributions of both horizontal and vertical components of the heat budgets. Thus, our observations are useful to confirm the assumption made in other studies (e.g., Chemke et al. 2020) that the horizontal component alone is useful to estimate advection; however, we note that it will generally provide an overestimate, due to the compensating nature of the vertical fluxes.

This study has also clearly demonstrated the importance of the depth of integration chosen to define the upper ocean. Previous studies have used the wintertime climatological MLD as the bottom boundary (Buckley et al. 2014, 2015) while other studies used a fixed depth horizon (e.g., Doney et al. 2007; Grist et al. 2010; Piecuch et al. 2017; Small et al. 2020). As we have shown, there are substantial differences in the spatial patterns of the covariance ratios between the heat budget terms when determined by integrating over a fixed depth versus when determined by integrating over the winter MLD. A striking example is given by the forcing-dominated subtropical regions (regions B2-B4 in Figure S9). This is consistent with previous studies that showed that $F_{\text {forc }}{ }^{\prime}$ dominates in the subtropical interior (Buckley et al. 2014, 2015). However, we show that the 
dominance of $F_{\text {forc }}{ }^{\prime}$ vanishes simply by integrating over the upper $300 \mathrm{~m}$ (Figure 8, Figure S12, S13). These results show that globally integrating the heat budget over a fixed depth should be understood with the caveat that different ocean regions cannot be easily compared because of the large spatial variation in the extent of wintertime mixing (Figure S1). Therefore, integrating over a fixed-depth layer will affect the balance in the heat budget in different ways depending on the relationship between that fixed-layer depth and the depth of the wintertime climatological MLD.

For shallower layers (i.e., upper $50-100 \mathrm{~m})$ the balance between $F_{\text {forc }}{ }^{\prime}$ and $-\nabla \cdot(\mathbf{u} \theta)^{\prime}$ is comparable to the one determined over the winter MLD. With increasing depths of integration, the balance between $F_{\text {forc }}{ }^{\prime}$ and $-\nabla \cdot(\mathbf{u} \theta)^{\prime}$ shifts towards higher contribution of the advective terms. The contribution of $F_{\text {forc }}$ is generally greater at shallower layers (i.e., upper 50-100 m) as it is represented mostly by solar radiation and heat exchange at the air-sea interface. As the depth of integration increases, $-\nabla \cdot(\mathbf{u} \theta)^{\prime}$ becomes more important and forcing diminishes, in the lower latitudes. When integrating over the entire water column, $F_{\text {forc }}{ }^{\prime}$ remains relevant only in the higher latitudes as a result of the deep winter MLD there.

The heat budget is also sensitive to the temporal scale. Averaging over longer time intervals (i.e., varying the temporal mean from monthly to decadal), results in a decrease in $F_{\text {forc }}{ }^{\prime}$ as the major contributor, concomitant with an increase in the contribution by $-\nabla \cdot(\mathbf{u} \theta)^{\prime}$. This suggests that heat content variability is largely forcing-driven on shorter time scales, while advective processes are increasingly important at longer time scales. Such time scale dependencies have been reported for the North Atlantic by Buckley et al. $(2014,2015)$ and for the WBCs and ACC by Bishop et al. (2017). We have shown here that this transition from forcing to advection-driven budgets as temporal aggregation increases is common in most dynamical regimes. Thus, for future studies, it is important to clearly define at what temporal scales heat content variability is analysed. 
Interestingly, it is mostly $-\nabla\left(\overline{\mathbf{u}}^{m} \theta^{\prime}\right)$ that becomes dominant at longer time scales. The greater importance of mean advection of anomalous heat content at long time scales is consistent with studies which treat the long-term ocean-heat-uptake problem as a passive tracer transport phenomenon (Zanna et al. 2019). The spatial pattern of covariance ratios we have described in this study is compatible with the conclusion from Armour et al. (2016), who studied the effect of mean circulation on temperature trends in the Southern Ocean. They conclude that south of the ACC, mean circulation is responsible for the relatively weak SST trends. We also find that $-\nabla\left(\overline{\mathbf{u}}^{m} \theta^{\prime}\right)$ is the dominant driver of temperature variability in the Southern Ocean at longer time scales (Figures 4 ) and that $F_{\text {forc }}{ }^{\prime}$ plays a lesser role here. This is in contrast to the high latitudes of the Northern Hemisphere, where we find $F_{\text {forc }}{ }^{\prime}$ to be more dominant.

Consistent with recent studies by Bishop et al. (2017) and Small et al. (2019, 2020), we find that spatial aggregation of the gridded ECCOv4 fields to coarser resolutions changes the balance between forcing and advection. However, in our case the overall patterns remain the same when aggregating the grid from the original resolution of $1^{\circ} \times 1^{\circ}$ up to a factor of 90 . This low sensitivity of the heat budget to aggregation scale is surprising, as the expectation would be that the balance of mechanisms in the budget would shift more substantially towards $F_{\text {forc }}{ }^{\prime}$ as aggregation occurs over larger scales. However, only a moderate increase in the contribution of $F_{\text {forc }}{ }^{\prime}$ was observed as the spatial scale coarsened, such that $F_{\text {forc }}{ }^{\prime}$ is dominant only at the major basin to global scale. Similarly, the contribution by $-\nabla \cdot(\mathbf{u} \theta)^{\prime}$ decreases only slightly with coarsening, mostly in the high latitudes. Advection remains the main contributor in the low latitudes, even at the largest aggregation scale (i.e., $90 \times 90$ ). Note that the focus on spatial scale dependence is for zonal means, not specific regions such as the WBC extensions and $\mathrm{ACC}$, which was beyond the scope of the study. In any case, the likely reason for the difference from previous studies (Bishop et al. 2017; Small et al. 2019, 2020) is that the spatial resolution of the ECCOv4 state estimate is already 
too coarse to resolve mesoscale dynamics. The only possible exception is for the tropical oceans, where the advective-driven signal occurs on such a large scale that it is resolved in ECCOv4.

The highest $n$ value $(n=90)$ corresponds to approximately $90^{\circ} \times 90^{\circ}$, which can be considered a basin-wide scale. Any coarser aggregation would lead to summing over different ocean basins (across continents) which would yield ambivalent results in terms of potential underlying mechanisms. Coarsening of the grid beyond the $90^{\circ} \times 90^{\circ}$ was addressed by evaluating the heat budget for the three major ocean basins (Pacific, Atlantic, Indian). With this analysis we clearly show that advection remains relevant for large parts of the ocean at basin-wide scales. Thus, it is not possible to determine a specific resolution scale at which point $-\nabla \cdot(\mathbf{u} \theta)^{\prime}$ will become zero. Instead it should be recognized that advective processes only become secondary when integrating over the major ocean basins. However even then the dominance of $-\nabla \cdot(\mathbf{u} \theta)^{\prime}$ at longer time scales is evident (Figure S6). In the case of basin-integrated upper ocean heat budget analysis, there is only secondary influence through horizontal exchanges between the basins, and instead vertical heat fluxes contribute considerably. Thus, only for the global and full-depth integral can the advective terms be neglected.

We note certain caveats associated with our study. First and foremost, ECCOv4 is a relatively coarse resolution model and therefore unable to resolve mesoscale ocean processes. Similar to the work presented here, Small et al. (2020) evaluated ocean heat budgets over the upper $50 \mathrm{~m}$ and $400 \mathrm{~m}$, using both a high- and low-resolution setup. An important insight regarding the impact of resolution arose when performing spatial smoothing with their high-resolution model output to determine at what scale the high-resolution model results reflects the low-resolution results. They found that for most regions this occurs when averaging over a box of $3^{\circ}$ to $5^{\circ}$ for the $50 \mathrm{~m}$ budget and $5^{\circ}$ to $7^{\circ}$ for the $400 \mathrm{~m}$ budget. As most of the sensitivity to spatial resolution lies below $1^{\circ}$ (Bishop 
et al. 2017; Small et al. 2020), it makes sense that the spatial aggregation with ECCOv4 did not lead to large differences globally, as the spatial resolution of ECCOv4 is around $1^{\circ}$.

While higher spatial resolution is important in capturing ocean dynamics relevant to heat content variability, it is currently not feasible in a reanalysis framework to present estimates at resolutions below $1^{\circ}$ and still ensure constraining them to available observations. Despite these limitations, ECCOv4 presents a distinct advantage in that it is a physically consistent estimate of the observed ocean state. It accurately reflects the ocean variability over larger regions, though it must be recognized that once the spatial resolution is increased, variability in mesoscale ocean dynamics will likely play a more important role in characterizing overall variability.

Another caveat of our approach is that only 24 years of data are available, limiting our capability to analyze the budget on a decadal time scale. The issue that arises is that at longer temporal aggregation scales, the time series have fewer and fewer points and so the correlations become more noisy. Thus, with the pentad averages the number of data points may be too small to yield robust results. On the other hand, our results are consistent with the findings of other studies (Buckley et al. 2014, 2015; Bishop et al. 2017). By using multiple temporal aggregations we were able to reveal a clear shift towards advective-driven heat budgets which often occurs at particular time scales. For most dynamic regions this was shown with averaging beyond a 2-year time scale. We encourage the application of our time aggregation methodology to longer dataset runs (e.g, hindcast simulations or coupled-climate models), in order to provide an independent and more robust way to identify important time scales at which shifts in the heat budget balance can be expected.

Data availability statement. All results of this study are based on ECCO Version 4, Release 3 (ECCOv4r3) for which standard output and documentation can be obtained at https://ecco. 
jpl.nasa.gov/drive/files/Version4/Release3/. We reproduced the ECCOv4r3 ocean state estimate with a custom set of diagnostics which are available as a dataset on Pangeo (http: //catalog.pangeo.io/ocean/ECCOv4r3) or can be requested from the corresponding author.

Acknowledgments. JET acknowledges funding from NASA's Goddard Space Flight Center (Award NNX15AN27H). RPA acknowledges support from NSF Award OCE-1553593 and a Sloan Fellowship in Ocean Sciences. Computational tools for performing this research were provided by Pangeo, supported by NSF EarthCube award OCE-1740648. The authors thank Spencer Jones for providing helpful comments. We wish to thank Martha Buckley and two anonymous reviewers for their careful assessment of the manuscript and for their helpful suggestions on improving it.

\section{References}

Adcroft, A., and J.-M. Campin, 2004: Rescaled height coordinates for accurate representation of free-surface flows in ocean circulation models. Ocean Modelling, 7 (3), 269-284, doi:10.1016/ j.ocemod.2003.09.003.

Alexander, M. A., and C. Deser, 1995: A Mechanism for the Recurrence of Wintertime Midlatitude SST Anomalies. Journal of Physical Oceanography, 25 (1), 122-137, doi: 10.1175/1520-0485(1995)025<0122:AMFTRO>2.0.CO;2.

Ardyna, M., H. Claustre, J.-B. Sallée, F. D’Ovidio, B. Gentili, G. van Dijken, F. D’Ortenzio, and K. R. Arrigo, 2017: Delineating environmental control of phytoplankton biomass and phenology in the Southern Ocean. Geophysical Research Letters, 44 (10), 5016-5024, doi: 10.1002/2016GL072428.

Armour, K. C., J. Marshall, J. R. Scott, A. Donohoe, and E. R. Newsom, 2016: Southern Ocean warming delayed by circumpolar upwelling and equatorward transport. Nature Geoscience, 9 (7), 
Barnett, T. P., D. W. Pierce, K. M. AchutaRao, P. J. Gleckler, B. D. Santer, J. M. Gregory, and W. M. Washington, 2005: Penetration of Human-Induced Warming into the World's Oceans. Science, 309 (5732), 284-287, doi:10.1126/science.1112418.

Barnett, T. P., D. W. Pierce, and R. Schnur, 2001: Detection of Anthropogenic Climate Change in the World's Oceans. Science, 292 (5515), 270-274, doi:10.1126/science.1058304.

Bigg, G. R., T. D. Jickells, P. S. Liss, and T. J. Osborn, 2003: The role of the oceans in climate. International Journal of Climatology, 23 (10), 1127-1159, doi:10.1002/joc.926.

Bishop, S. P., R. J. Small, F. O. Bryan, and R. A. Tomas, 2017: Scale Dependence of Midlatitude Air-Sea Interaction. Journal of Climate, 30 (20), 8207-8221.

Buckley, M. W., R. M. Ponte, G. Forget, and P. Heimbach, 2014: Low-Frequency SST and UpperOcean Heat Content Variability in the North Atlantic. Journal of Climate, 27 (13), 4996-5018, doi:10.1175/JCLI-D-13-00316.1.

Buckley, M. W., R. M. Ponte, G. Forget, and P. Heimbach, 2015: Determining the Origins of Advective Heat Transport Convergence Variability in the North Atlantic. Journal of Climate, 28 (10), 3943-3956, doi:10.1175/JCLI-D-14-00579.1.

Campin, J.-M., A. Adcroft, C. Hill, and J. Marshall, 2004: Conservation of properties in a freesurface model. Ocean Modelling, 6 (3), 221-244, doi:10.1016/S1463-5003(03)00009-X.

Chemke, R., L. Zanna, and L. M. Polvani, 2020: Identifying a human signal in the North Atlantic warming hole. Nature Communications, 11 (1540), 1-7, doi:10.1038/s41467-020-15285-x.

Devred, E., S. Sathyendranath, and T. Platt, 2007: Delineation of ecological provinces using ocean colour radiometry. Marine Ecology Progress Series, 346, 1-13, doi:10.3354/meps07149. 
Doney, S. C., S. Yeager, G. Danabasoglu, W. G. Large, and J. C. McWilliams, 2007: Mechanisms Governing Interannual Variability of Upper-Ocean Temperature in a Global Ocean Hindcast Simulation. Journal of Physical Oceanography, 37 (7), 1918-1938.

Forget, G., J.-M. Campin, P. Heimbach, C. N. Hill, R. M. Ponte, and C. Wunsch, 2015: ECCO version 4: an integrated framework for non-linear inverse modeling and global ocean state estimation. Geoscientific Model Development, 8 (10), 3071-3104, doi:10.5194/gmd-8-3071-2015.

Gong, X., and M. B. Richman, 1995: On the Application of Cluster Analysis to Growing Season Precipitation Data in North America East of the Rockies. Journal of Climate, 8 (4), 897-931, doi:10.1175/1520-0442(1995)008<0897:OTAOCA>2.0.CO;2.

Grist, J.P., and Coauthors, 2010: The roles of surface heat flux and ocean heat transport convergence in determining Atlantic Ocean temperature variability. Ocean Dynamics, 60 (4), 771-790.

Hartigan, J. A., and M. Wong, 1979: Algorithm AS 136: A k-means clustering algorithm. Journal of the Royal Statistical Society. Series C Applied Statistics, 28 (1), 100-108, doi: $10.2307 / 2346830$.

Hasselmann, K., 1976: Stochastic climate models, Part I. Theory. Tellus, 28 (6), 473-485, doi: 10.1111/j.2153-3490.1976.tb00696.x.

Hill, C., D. Ferreira, J.-M. Campin, J. Marshall, R. Abernathey, and N. Barrier, 2012: Controlling spurious diapycnal mixing in eddy-resolving height-coordinate ocean models - Insights from virtual deliberate tracer release experiments. Ocean Modelling, 45, 14-26, doi: 10.1016/j.ocemod.2011.12.001. 
Keenlyside, N. S., M. Latif, J. Jungclaus, L. Kornblueh, and E. Roeckner, 2008: Advancing decadal-scale climate prediction in the North Atlantic sector. Nature, 453, 84-88, doi:10.1038/ nature06921.

Kirtman, B. P., and Coauthors, 2012: Impact of ocean model resolution on CCSM climate simulations. Climate Dynamics, 39 (6), 1303-1328, doi:10.1007/s00382-012-1500-3.

Lee, S.-K., W. Park, E. van Sebille, M. O. Baringer, C. Wang, D. B. Enfield, S. G. Yeager, and B. P. Kirtman, 2011: What caused the significant increase in Atlantic Ocean heat content since the mid-20th century? Geophysical Research Letters, 38 (17), doi:10.1029/2011GL048856.

Liang, X., C. Wunsch, P. Heimbach, and G. Forget, 2015: Vertical Redistribution of Oceanic Heat Content. Journal of Climate, 28 (9), 3821-3833, doi:10.1175/JCLI-D-14-00550.1.

Liu, W., and S.-P. Xie, 2018: An Ocean View of the Global Surface Warming Hiatus. Oceanography, 31 (2), 72-79, doi:10.5670/oceanog.2018.217.

Lund, R., and B. Li, 2009: Revisiting Climate Region Definitions via Clustering. Journal of Climate, 22, 1787-1800, doi:10.1175/2008JCLI2455.1.

Megann, A., 2018: Estimating the numerical diapycnal mixing in an eddy-permitting ocean model. Ocean Modelling, 121, 19-33, doi:10.1016/j.ocemod.2017.11.001.

Piecuch, C. G., and R. M. Ponte, 2012: Importance of Circulation Changes to Atlantic Heat Storage Rates on Seasonal and Interannual Time Scales. Journal of Climate, 25 (1), 350-362, doi:10.1175/JCLI-D-11-00123.1.

Piecuch, C. G., R. M. Ponte, C. M. Little, M. W. Buckley, and I. Fukumori, 2017: Mechanisms underlying recent decadal changes in subpolar North Atlantic Ocean heat content. Journal of Geophysical Research: Oceans, 122 (9), 7181-7197, doi:10.1002/2017JC012845. 
Pierce, D. W., P. J. Gleckler, T. P. Barnett, B. D. Santer, and P. J. Durack, 2012: The fingerprint of human-induced changes in the ocean's salinity and temperature fields. Geophysical Research Letters, 39 (21), doi:10.1029/2012GL053389.

Roberts, C. D., D. Calvert, N. Dunstone, L. Hermanson, M. D. Palmer, and D. Smith, 2016: On the Drivers and Predictability of Seasonal-to-Interannual Variations in Regional Sea Level. Journal of Climate, 29 (21), 7565-7585, doi:10.1175/JCLI-D-15-0886.1.

Roberts, C. D., M. D. Palmer, R. P. Allan, D. G. Desbruyeres, P. Hyder, C. Liu, and D. Smith, 2017: Surface flux and ocean heat transport convergence contributions to seasonal and interannual variations of ocean heat content. Journal of Geophysical Research: Oceans, 122 (1), 726-744, doi:10.1002/2016JC012278.

Robson, J. I., R. T. Sutton, and D. M. Smith, 2012: Initialized decadal predictions of the rapid warming of the North Atlantic Ocean in the mid 1990s. Geophysical Research Letters, 39 (19), doi:10.1029/2012GL053370.

Small, R. J., F. O. Bryan, S. P. Bishop, S. Larson, and R. A. Tomas, 2020: What drives upper ocean temperature variability in coupled climate models and observations? Journal of Climate, 33 (2), 577-596, doi:10.1175/JCLI-D-19-0295.1.

Small, R. J., R. A. Tomas, F. O. Bryan, and S. P. Bishop, 2019: Air-sea turbulent heat fluxes in climate models and observational analyses: what drives their variability? Journal of Climate, 32 (8), 2397-2421, doi:10.1175/JCLI-D-18-0576.1.

Sonnewald, M., S. Dutkiewicz, C. Hill, and G. Forget, 2020: Elucidating ecological complexity: Unsupervised learning determines global marine eco-provinces. Science Advances, 6 (22), eaay4740, doi:10.1126/sciadv.aay4740. 
Sonnewald, M., C. Wunsch, and P. Heimbach, 2019: Unsupervised Learning Reveals Geography of Global Ocean Dynamical Regions. Earth and Space Science, 6, 784-794, doi:10.1029/ 2018EA000519.

Trenberth, K. E., J. T. Fasullo, and M. A. Balmaseda, 2014: Earth's Energy Imbalance. Journal of Climate, 27 (9), 3129-3144, doi:10.1175/JCLI-D-13-00294.1.

von Schuckmann, K., and Coauthors, 2016: An imperative to monitor Earth's energy imbalance. Nature Climate Change, 6, 138-144, doi:10.1038/nclimate2876.

von Storch, J.-S., 2000: Signatures of Air-Sea Interactions in a Coupled Atmosphere-Ocean GCM. Journal of Climate, 13 (1), 3361-3379, doi:10.1175/1520-0442(2000)013<3361:SOASII>2.0. $\mathrm{CO} ; 2$

Warren, B. A., 1999: Approximating the energy transport across oceanic sections. Journal of Geophysical Research: Oceans, 104 (C), 7915-7919, doi:10.1029/1998JC900089.

Wu, R., B. P. Kirtman, and K. Pegion, 2006: Local Air-Sea Relationship in Observations and Model Simulations. Journal of Climate, 19 (19), 4914-4932, doi:10.1175/JCLI3904.1.

Yan, X.-H., T. Boyer, K. Trenberth, T. R. Karl, S.-P. Xie, V. Nieves, K.-K. Tung, and D. Roemmich, 2016: The global warming hiatus: Slowdown or redistribution? Earth's Future, 4(11), 472-482, doi:10.1002/2016EF000417.

Yeager, S., A. Karspeck, G. Danabasoglu, J. Tribbia, and H. Teng, 2012: A decadal prediction case study: Late twentieth-century North Atlantic Ocean heat content. Journal of Climate, 25 (15), 5173-5189, doi:10.1175/JCLI-D-11-00595.1. 
Zanna, L., S. Khatiwala, J. M. Gregory, J. Ison, and P. Heimbach, 2019: Global reconstruction of historical ocean heat storage and transport. Proceedings of the National Academy of Sciences, 116 (4), 1126-1131, doi:10.1073/pnas.1808838115.

Zhang, R., 2017: On the persistence and coherence of subpolar sea surface temperature and salinity anomalies associated with the Atlantic multidecadal variability. Geophysical Research Letters, 44 (15), 7865-7875, doi:10.1002/2017GL074342. 
Table 1. Global average covariance ratios for heat budget terms at different spatial aggregations. Monthly heat budget terms were integrated over the wintertime climatological MLD. The aggregation value refers to the level of binning, where $n \times n$ aggregation indicates grouping of $n$ grid cells along both $x$ and $y$ axes in the horizontal space. 
TABLE 1. Global average covariance ratios for heat budget terms at different spatial aggregations. Monthly heat budget terms were integrated over the wintertime climatological MLD. The aggregation value refers to the level of binning, where $n \times n$ aggregation indicates grouping of $n$ grid cells along both $x$ and $y$ axes in the horizontal space.

\begin{tabular}{lccccccc}
\hline Aggregation & $F_{\text {forc }}^{\prime}$ & $-\nabla \cdot(\mathbf{u} \theta)^{\prime}$ & $-\nabla \cdot \mathbf{F}_{\text {diff }}$ & $-\nabla \cdot\left(\mathbf{u}^{\prime} \bar{\theta}^{m}\right)$ & $-\nabla \cdot\left(\overline{\mathbf{u}}^{m} \theta^{\prime}\right)$ & $-\nabla \cdot\left(\mathbf{u}^{\prime} \theta^{\prime}-\overline{\mathbf{u}^{\prime} \theta^{\prime}}{ }^{m}\right)$ & $\mathrm{R}$ \\
\hline $1 \times 1$ & 0.55 & 0.44 & 0.00 & 0.31 & 0.10 & 0.03 & 0.01 \\
$2 \times 2$ & 0.56 & 0.43 & 0.00 & 0.30 & 0.10 & 0.03 & 0.01 \\
$3 \times 3$ & 0.58 & 0.42 & -0.00 & 0.30 & 0.09 & 0.03 & 0.01 \\
$5 \times 5$ & 0.61 & 0.39 & -0.00 & 0.28 & 0.08 & 0.02 & 0.01 \\
$6 \times 6$ & 0.62 & 0.38 & -0.00 & 0.28 & 0.08 & 0.02 & 0.01 \\
$9 \times 9$ & 0.66 & 0.34 & -0.01 & 0.26 & 0.07 & 0.02 & 0.00 \\
$10 \times 10$ & 0.66 & 0.34 & -0.01 & 0.25 & 0.06 & 0.02 & 0.00 \\
$15 \times 15$ & 0.70 & 0.30 & -0.01 & 0.23 & 0.05 & 0.02 & 0.00 \\
$18 \times 18$ & 0.71 & 0.29 & -0.01 & 0.22 & 0.05 & 0.02 & 0.00 \\
$30 \times 30$ & 0.76 & 0.25 & -0.01 & 0.20 & 0.04 & 0.02 & 0.00 \\
$45 \times 45$ & 0.74 & 0.27 & -0.02 & 0.21 & 0.04 & 0.02 & 0.00 \\
$90 \times 90$ & 0.70 & 0.30 & -0.00 & 0.21 & 0.06 & 0.03 & 0.01 \\
\hline
\end{tabular}




\section{LIST OF FIGURES}

Fig. 1. Global distribution of the covariance ratios between the total tendency and anomalous (a) forcing $\left(F_{\text {forc }}{ }^{\prime}\right)$, (b) advection $\left(-\nabla \cdot(\mathbf{u} \theta)^{\prime}\right)$, (c) anomalous advection of the mean temperature field $\left(-\nabla\left(\mathbf{u}^{\prime} \bar{\theta}^{m}\right)\right),(\mathrm{d})$ mean advection of the anomalous temperature field $\left(-\nabla\left(\overline{\mathbf{u}}^{m} \theta^{\prime}\right)\right)$, (e) covariation of anomalies of both velocity and temperature fields $\left(-\nabla \cdot\left(\mathbf{u}^{\prime} \theta^{\prime}-{\overline{\mathbf{u}^{\prime} \theta^{\prime}}}^{m}\right)\right)$ and (f) anomalous diffusion $\left(-\nabla \cdot \mathbf{F}_{\text {diff }}{ }^{\prime}\right)$. The terms are integrated over the climatological maximum MLD (i.e., winter MLD) and the covariance ratios have been evaluated on the original spatial $(1 \times 1)$ and temporal (monthly) resolutions.

Fig. 2. Global distribution of covariance ratios at different depths of integration. Each column represents the main budget terms (left to right): anomalous forcing $\left(F_{\text {forc }}{ }^{\prime}\right)$, anomalous advection of the mean temperature field $\left(-\nabla\left(\mathbf{u}^{\prime} \bar{\theta}^{m}\right)\right)$, mean advection of the anomalous temperature field $\left(-\nabla\left(\overline{\mathbf{u}}^{m} \theta^{\prime}\right)\right)$ and anomalous diffusion $\left(-\nabla \cdot \mathbf{F}_{\text {diff }}{ }^{\prime}\right)$. Each row represents the depth level over which budget terms are integrated (top to bottom): $50 \mathrm{~m}, 100 \mathrm{~m}, 300 \mathrm{~m}$, $700 \mathrm{~m}, 2000 \mathrm{~m}$ and $6000 \mathrm{~m}$ (i.e., full-depth). The covariance ratios have been evaluated on the original horizontal $(1 \times 1)$ and temporal (monthly) resolutions.

Fig. 3. Global distribution of the covariance ratios for different depths of integration. Each column represents following advective terms: anomalous horizontal advection of the mean temperature field, mean horizontal advection of the anomalous temperature field, anomalous vertical advection of the mean temperature field and mean vertical advection of the anomalous temperature field. Each row represents the depth level over which budget terms are integrated: winter MLD, $50 \mathrm{~m}, 100 \mathrm{~m}, 300 \mathrm{~m}, 700 \mathrm{~m}, 2000 \mathrm{~m}$ and $6000 \mathrm{~m}$ (i.e., full-depth). The covariance ratios have been evaluated on the original horizontal and temporal resolutions.

Fig. 4. Zonal means of the covariance ratios for the different budget terms in the upper ocean defined by winter MLD (top row), $300 \mathrm{~m}$ (center row) and $700 \mathrm{~m}$ (bottom row), and for monthly (left column), annual (middle column) and pentad (right column) temporal averages. Covariance ratios were derived from the original $(1 \times 1)$ spatial resolution and averaged into $10^{\circ}$ latitude bins.

Fig. 5. Zonal means of the covariance ratios for anomalous forcing $\left(F_{\text {forc }}{ }^{\prime}\right.$, blue lines $)$ and advection $\left(-\nabla \cdot(\mathbf{u} \theta)^{\prime}\right.$, red lines). Lines are shaded by spatial aggregation scale, with darker shades corresponding to coarser aggregations. Covariance ratios were derived from $F_{\text {forc }}{ }^{\prime}$ and $-\nabla \cdot(\mathbf{u} \theta)^{\prime}$ at each aggregation scale and averaged into $10^{\circ}$ latitude bins. Zonal means are presented for the upper ocean defined by winter MLD (top row), $300 \mathrm{~m}$ (center row) and $700 \mathrm{~m}$ (bottom row), as well as using monthly (left column), annual (middle column) and pentad (right column) temporal aggregation.

Fig. 6. Classifications of the ocean using k-means with five clusters labeled A to E, representing variation in dominance between forcing, advection and diffusion in the heat budget.

Fig. 7. Mean covariance ratios for the forcing $\left(F_{\text {forc }}{ }^{\prime}\right)$, advection $\left(-\nabla \cdot(\mathbf{u} \theta)^{\prime}\right)$ and diffusion $(-\nabla \cdot$ $\left.\mathbf{F}_{\text {diff }}{ }^{\prime}\right)$ terms in the anomaly heat budget for each cluster (A-E). The error bar denotes \pm 1 standard deviation.

Fig. 8. Covariance ratios for a selection of ocean regions at different integration depths $(50 \mathrm{~m}$, $100 \mathrm{~m}, 300 \mathrm{~m}, 700 \mathrm{~m}, 2000 \mathrm{~m}$ and $6000 \mathrm{~m})$ and time aggregation scales $(1 \mathrm{M}, 3 \mathrm{M}, 6 \mathrm{M}, 1 \mathrm{~A}$, 2A, 3A, 4A, 5A, 10A). Regions represent distinct oceanic regimes and are derived using k-means cluster analysis (Figure 5). Specific locations are shown in Figure S6. Each column represents the four heat budget terms (forcing, advection, diffusion, residual) for the specified 
region. Each panel sorts the covariance ratio for each term by integration depth along the vertical axis and time aggregation scale along the horizontal axis.

Fig. 9. Covariance ratio for a selection of ocean regions at different integration depths $(50 \mathrm{~m}, 100 \mathrm{~m}$, $300 \mathrm{~m}, 700 \mathrm{~m}, 2000 \mathrm{~m}$ and $6000 \mathrm{~m})$ and time aggregation scales (1M, 3M, 6M, 1A, 2A, 3A, 4A, 5A, 10A). Regions represent distinct oceanic regimes and are derived using kmeans cluster analysis (Figure 5). Specific locations are shown in Figure S6. Each column represents the decomposed terms for advection for the specified region. Each panel sorts the covariance ratio for each term by integration depth along the vertical axis and time aggregation scale along the horizontal axis. 

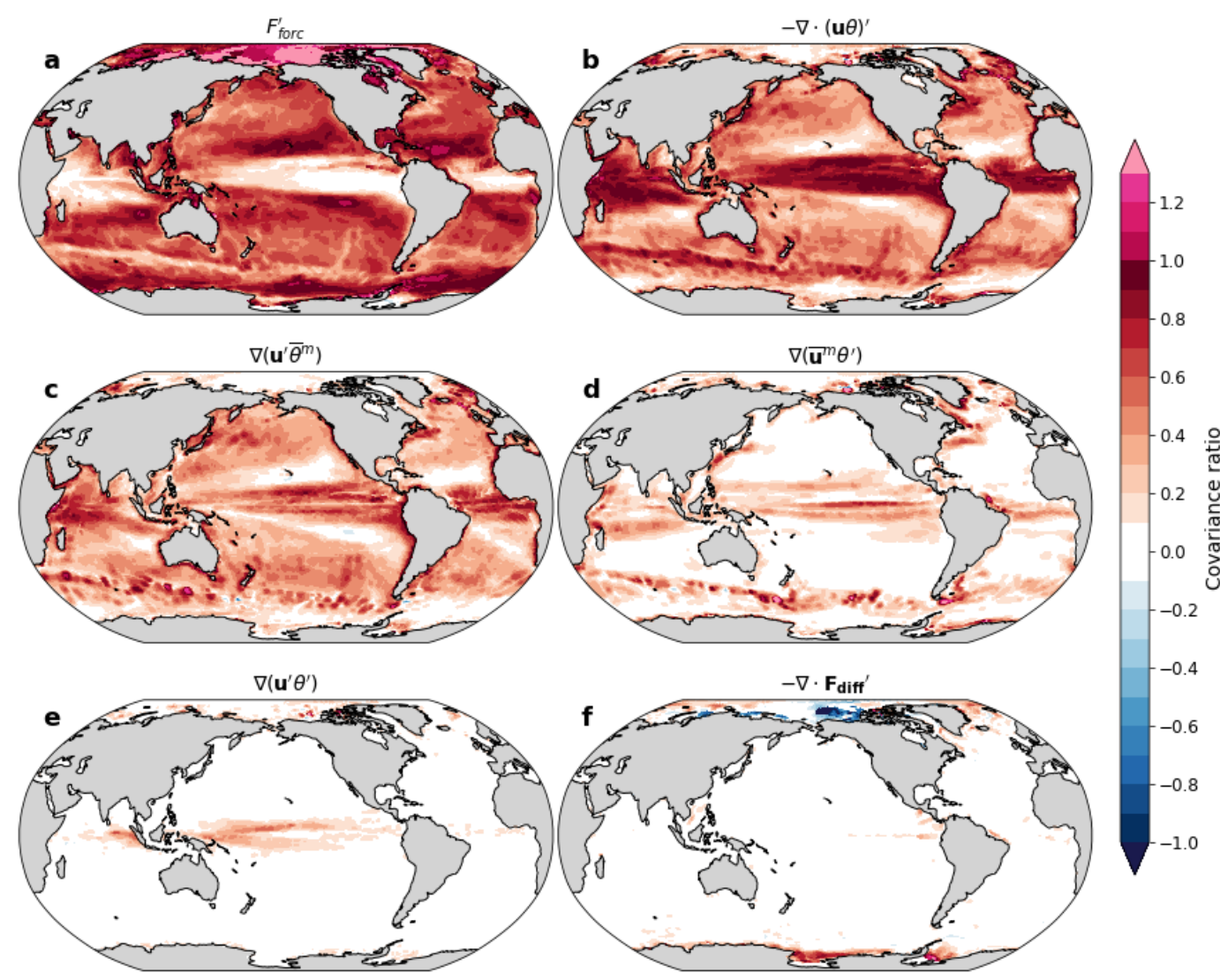

FIG. 1. Global distribution of the covariance ratios between the total tendency and anomalous (a) forcing $\left(F_{\text {forc }}{ }^{\prime}\right),\left(\right.$ b) advection $\left(-\nabla \cdot(\mathbf{u} \theta)^{\prime}\right),(\mathrm{c})$ anomalous advection of the mean temperature field $\left(-\nabla\left(\mathbf{u}^{\prime} \bar{\theta}^{m}\right)\right),(\mathrm{d})$ mean advection of the anomalous temperature field $\left(-\nabla\left(\overline{\mathbf{u}}^{m} \theta^{\prime}\right)\right)$, (e) covariation of anomalies of both velocity and temperature fields $\left(-\nabla \cdot\left(\mathbf{u}^{\prime} \theta^{\prime}-{\overline{\mathbf{u}^{\prime} \theta^{\prime}}}^{m}\right)\right)$ and (f) anomalous diffusion $\left(-\nabla \cdot \mathbf{F}_{\text {diff }}\right)$. The terms are integrated over the climatological maximum MLD (i.e., winter MLD) and the covariance ratios have been evaluated on the original spatial $(1 \times 1)$ and temporal (monthly) resolutions. 


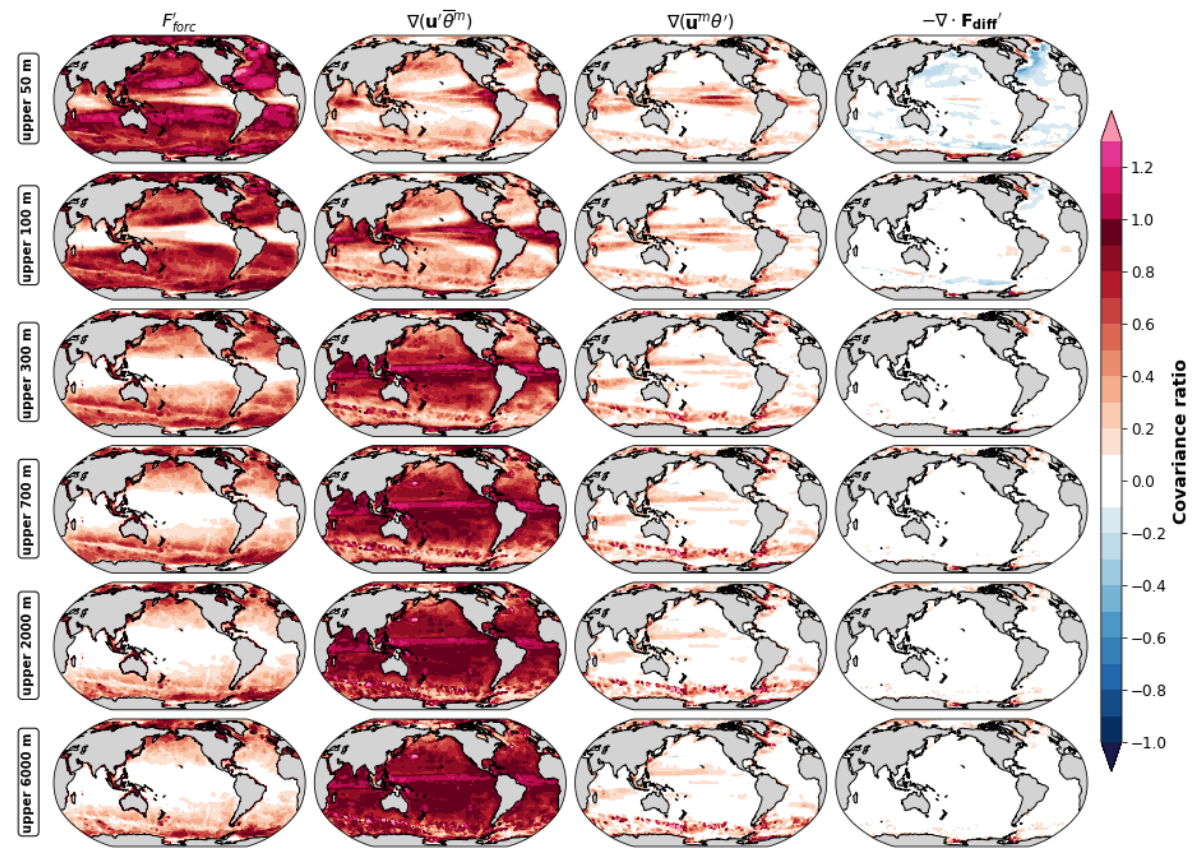

FIG. 2. Global distribution of covariance ratios at different depths of integration. Each column represents the main budget terms (left to right): anomalous forcing $\left(F_{\text {forc }}{ }^{\prime}\right)$, anomalous advection of the mean temperature field $\left(-\nabla\left(\mathbf{u}^{\prime} \bar{\theta}^{m}\right)\right)$, mean advection of the anomalous temperature field $\left(-\nabla\left(\overline{\mathbf{u}}^{m} \theta^{\prime}\right)\right)$ and anomalous diffusion $\left(-\nabla \cdot \mathbf{F}_{\mathrm{diff}}{ }^{\prime}\right)$. Each row represents the depth level over which budget terms are integrated (top to bottom): $50 \mathrm{~m}$, $100 \mathrm{~m}, 300 \mathrm{~m}, 700 \mathrm{~m}, 2000 \mathrm{~m}$ and $6000 \mathrm{~m}$ (i.e., full-depth). The covariance ratios have been evaluated on the original horizontal $(1 \times 1)$ and temporal (monthly) resolutions. 


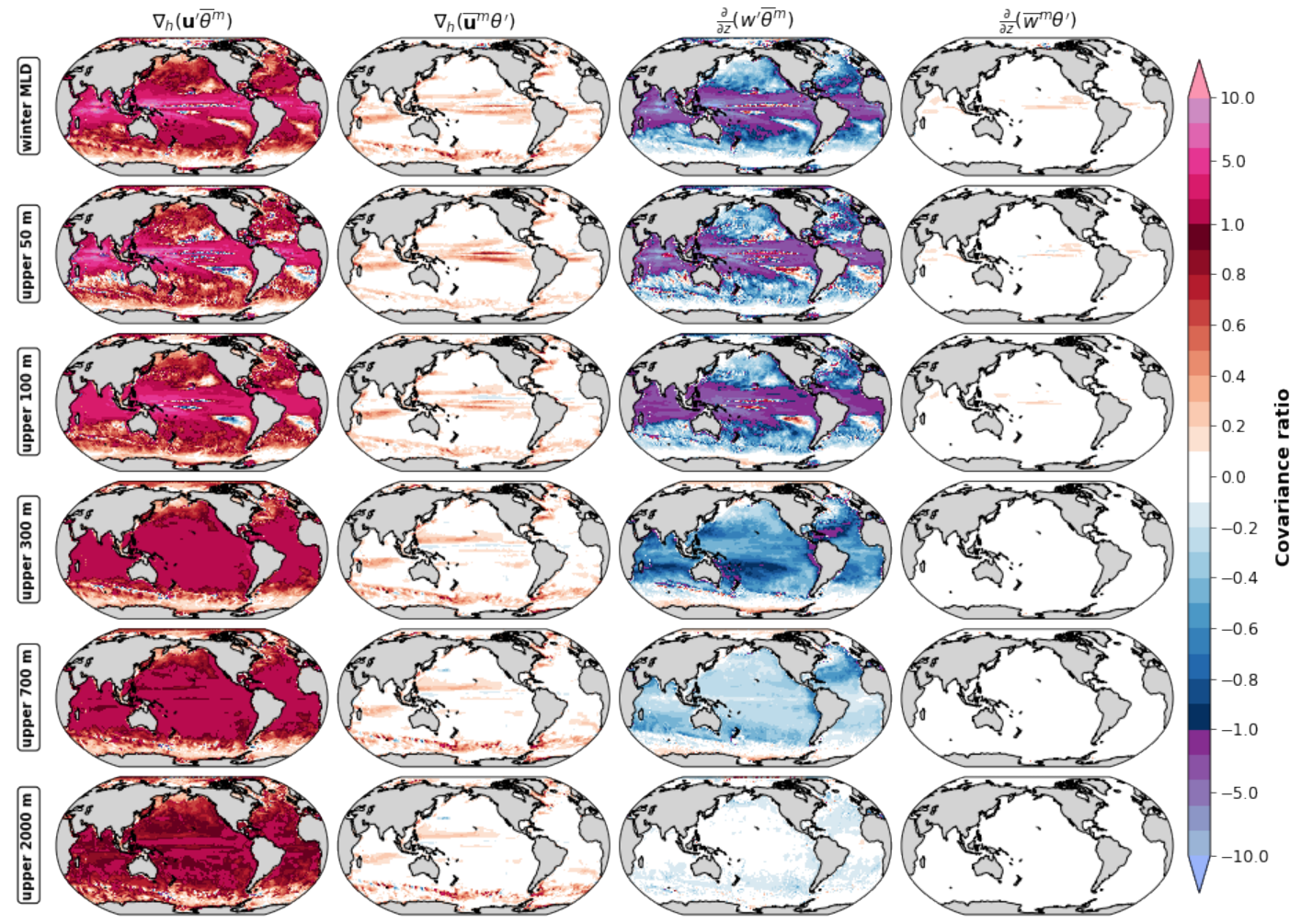

FIG. 3. Global distribution of the covariance ratios for different depths of integration. Each column represents following advective terms: anomalous horizontal advection of the mean temperature field, mean horizontal advection of the anomalous temperature field, anomalous vertical advection of the mean temperature field and mean vertical advection of the anomalous temperature field. Each row represents the depth level over which budget terms are integrated: winter MLD, 50 m, 100 m, 300 m, 700 m, 2000 m and 6000 m (i.e., full-depth). The covariance ratios have been evaluated on the original horizontal and temporal resolutions. 


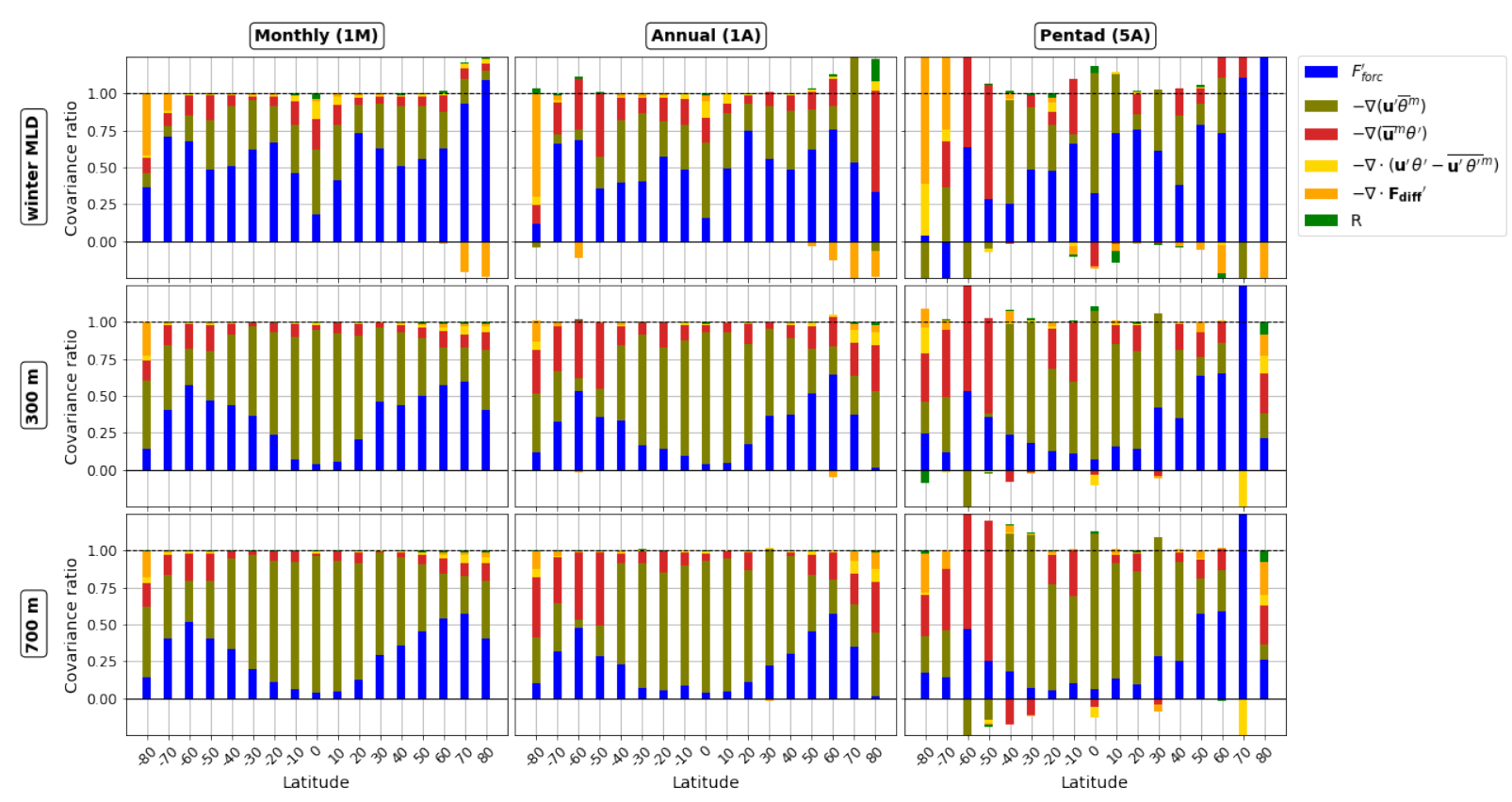

FIG. 4. Zonal means of the covariance ratios for the different budget terms in the upper ocean defined by winter MLD (top row), $300 \mathrm{~m}$ (center row) and $700 \mathrm{~m}$ (bottom row), and for monthly (left column), annual (middle column) and pentad (right column) temporal averages. Covariance ratios were derived from the original $(1 \times 1)$ spatial resolution and averaged into $10^{\circ}$ latitude bins. 


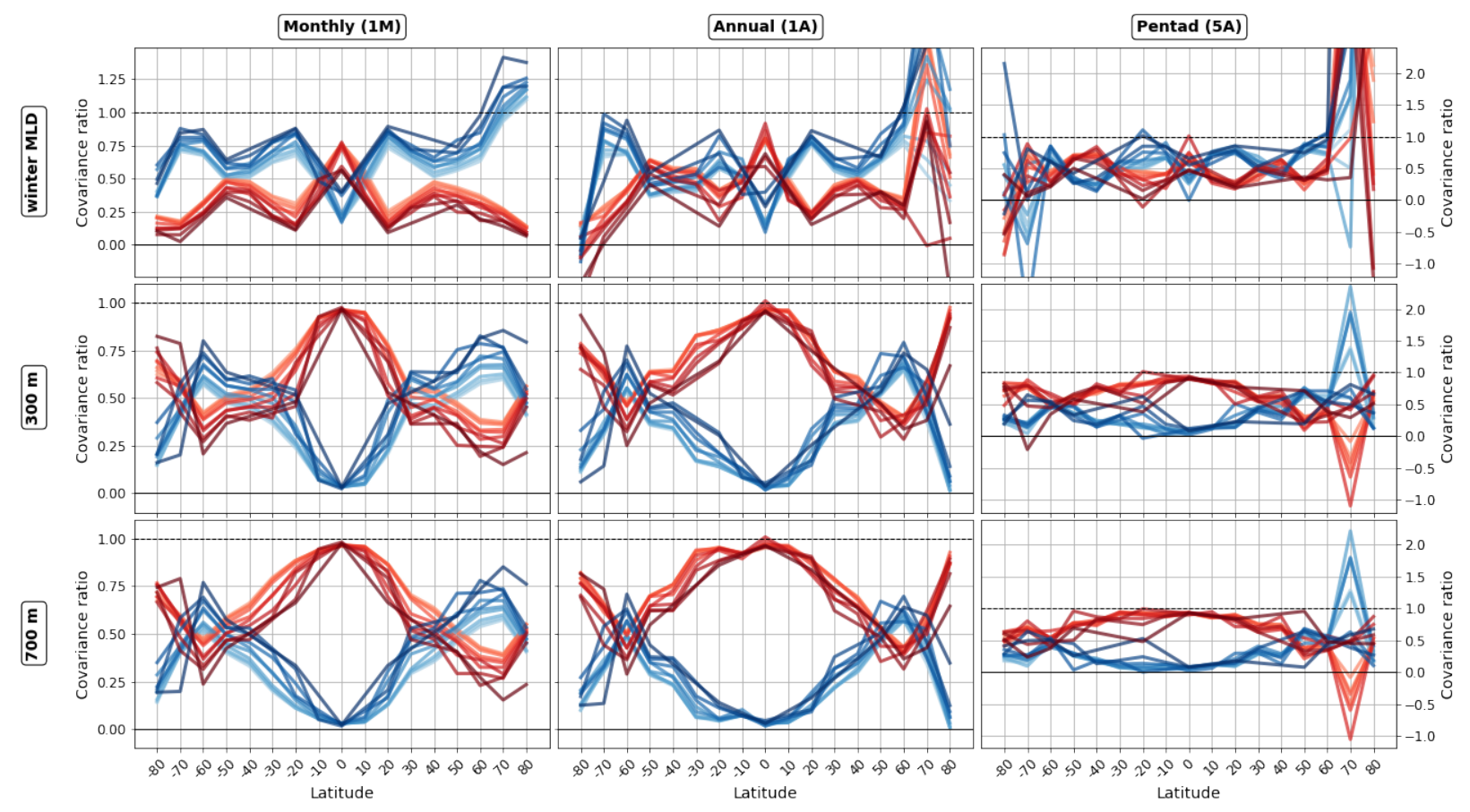

FIG. 5. Zonal means of the covariance ratios for anomalous forcing $\left(F_{\text {forc }}{ }^{\prime}\right.$, blue lines $)$ and advection $\left(-\nabla \cdot(\mathbf{u} \theta)^{\prime}\right.$, tions. Covariance ratios were derived from $F_{\text {forc }}{ }^{\prime}$ and $-\nabla \cdot(\mathbf{u} \theta)^{\prime}$ at each aggregation scale and averaged into $10^{\circ}$ latitude bins. Zonal means are presented for the upper ocean defined by winter MLD (top row), $300 \mathrm{~m}$ (center row) and $700 \mathrm{~m}$ (bottom row), as well as using monthly (left column), annual (middle column) and pentad (right column) temporal aggregation. 


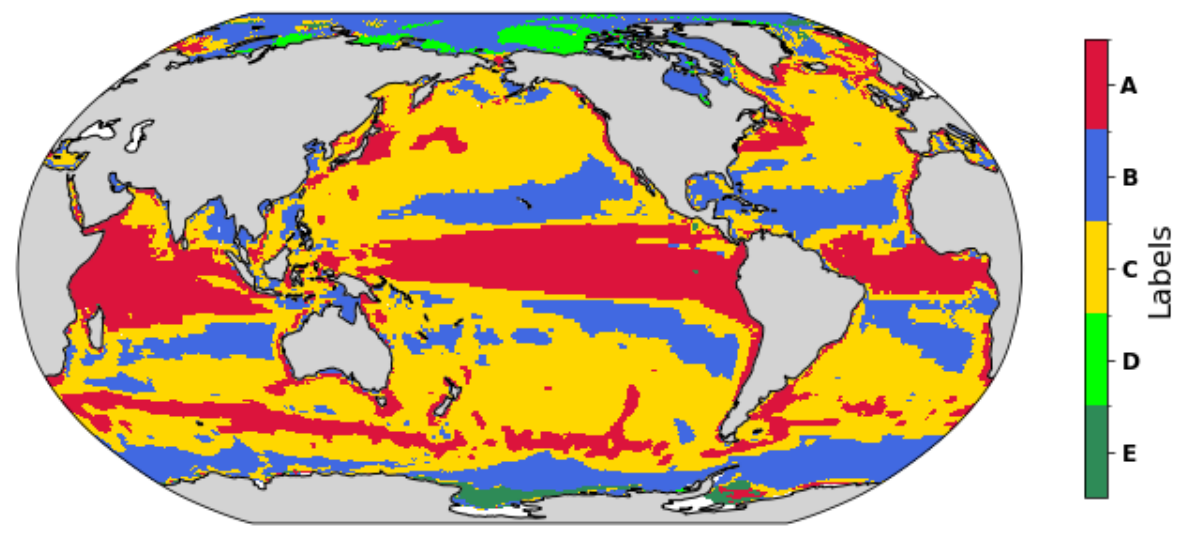

FIg. 6. Classifications of the ocean using k-means with five clusters labeled A to E, representing variation in dominance between forcing, advection and diffusion in the heat budget. 


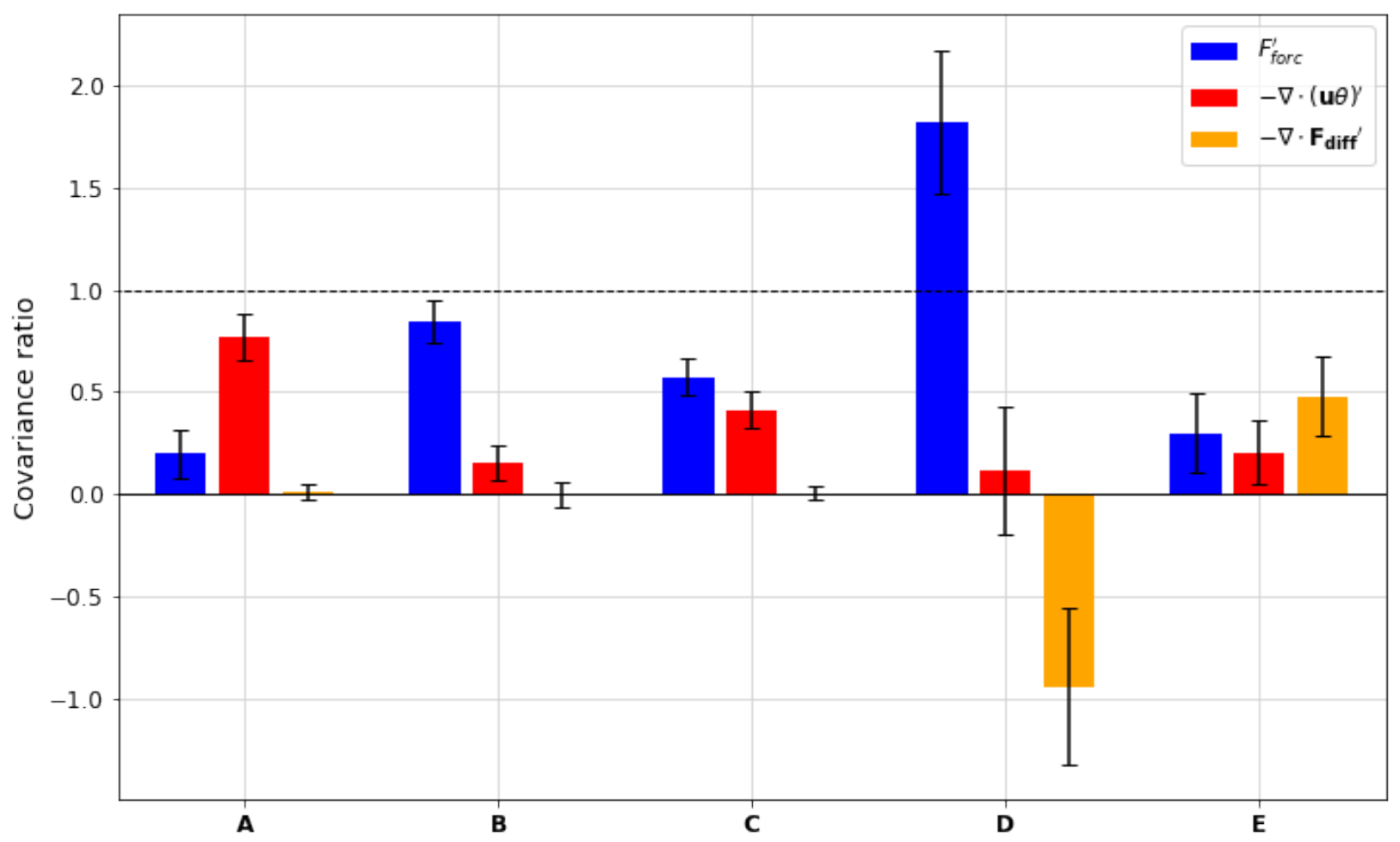

FIG. 7. Mean covariance ratios for the forcing $\left(F_{\text {forc }}{ }^{\prime}\right)$, advection $\left(-\nabla \cdot(\mathbf{u} \theta)^{\prime}\right)$ and diffusion $\left(-\nabla \cdot \mathbf{F}_{\text {diff }}{ }^{\prime}\right)$ terms in the anomaly heat budget for each cluster (A-E). The error bar denotes \pm 1 standard deviation. 


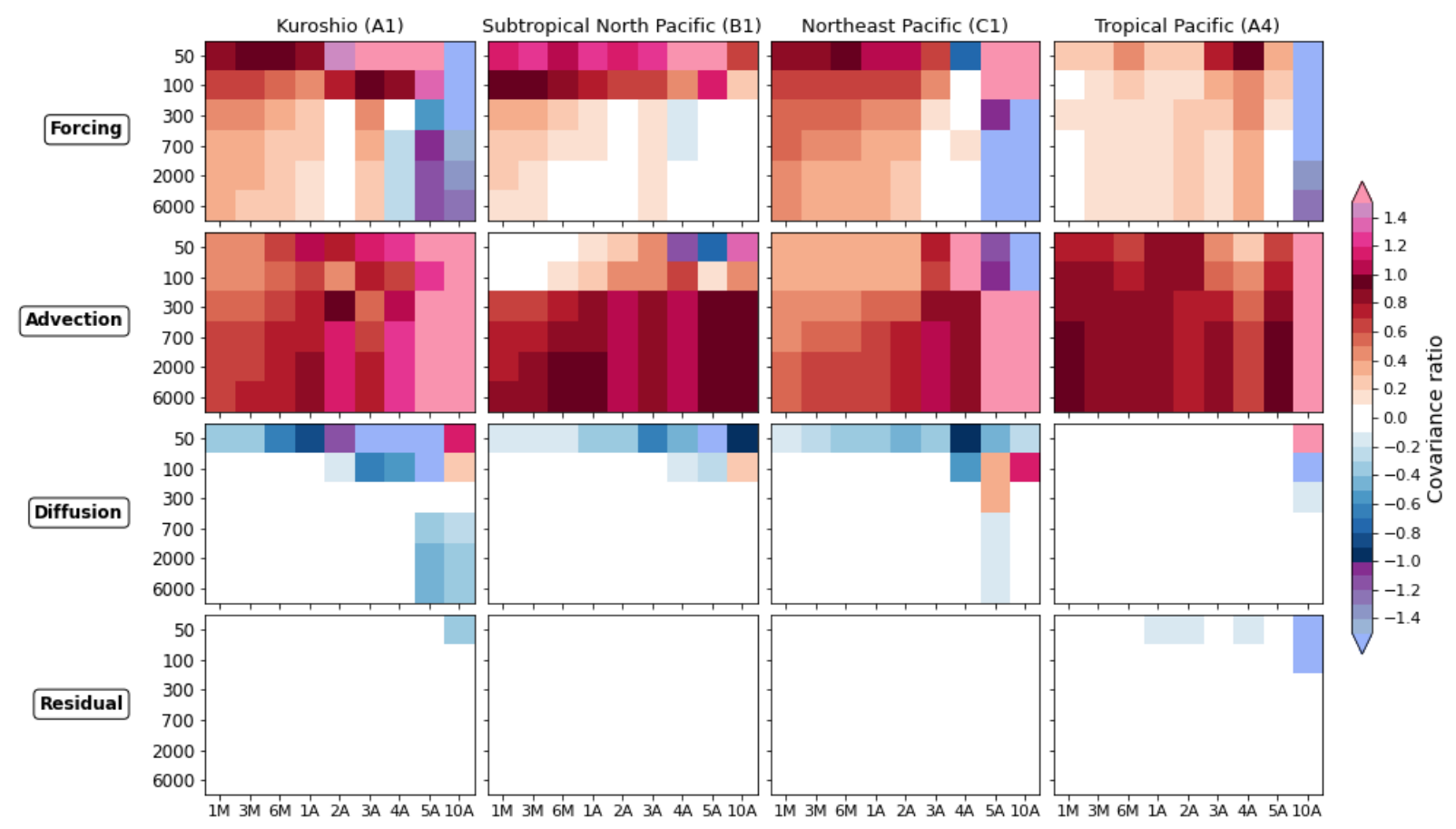

FIG. 8. Covariance ratios for a selection of ocean regions at different integration depths $(50 \mathrm{~m}, 100 \mathrm{~m}, 300 \mathrm{~m}$, $700 \mathrm{~m}, 2000 \mathrm{~m}$ and $6000 \mathrm{~m})$ and time aggregation scales (1M, 3M, 6M, 1A, 2A, 3A, 4A, 5A, 10A). Regions represent distinct oceanic regimes and are derived using k-means cluster analysis (Figure 5). Specific locations are shown in Figure S6. Each column represents the four heat budget terms (forcing, advection, diffusion, residual) for the specified region. Each panel sorts the covariance ratio for each term by integration depth along the vertical axis and time aggregation scale along the horizontal axis. 


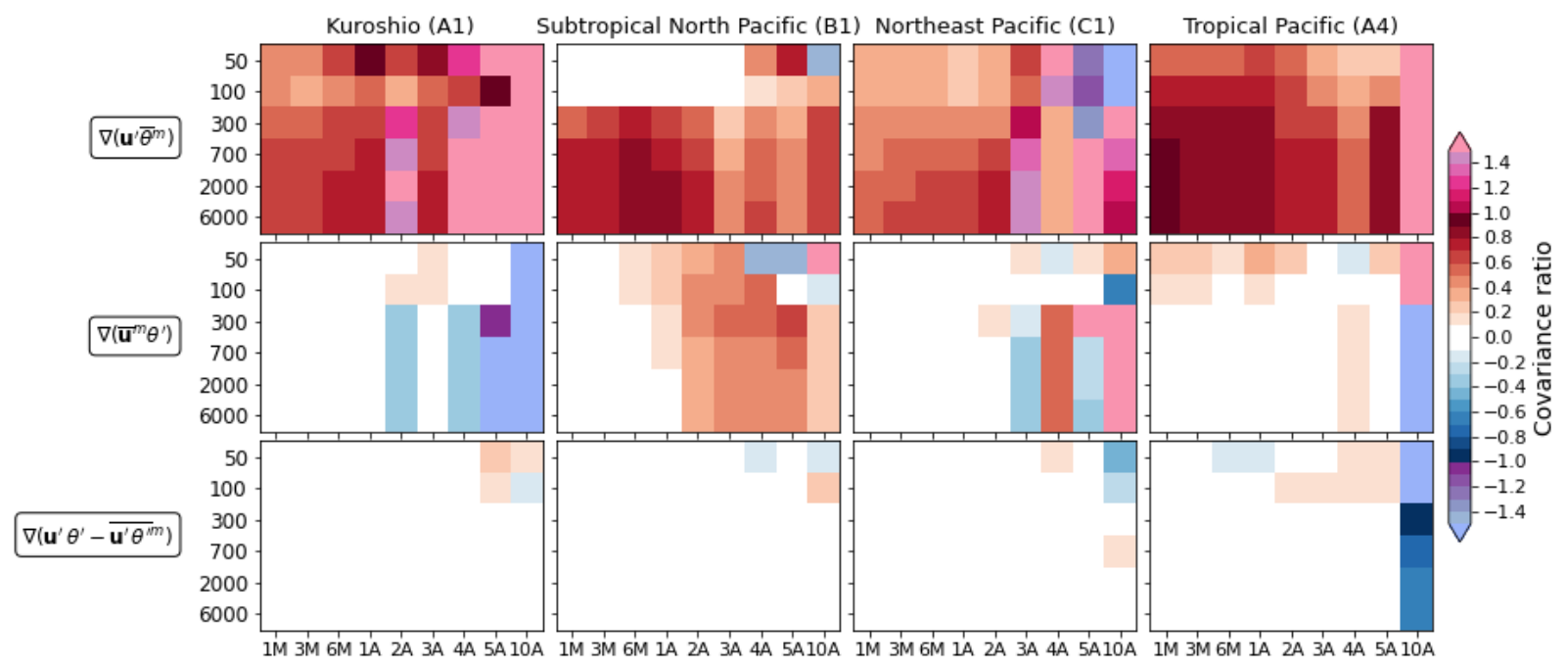

FIG. 9. Covariance ratio for a selection of ocean regions at different integration depths $(50 \mathrm{~m}, 100 \mathrm{~m}, 300 \mathrm{~m}$, $700 \mathrm{~m}, 2000 \mathrm{~m}$ and $6000 \mathrm{~m})$ and time aggregation scales (1M, 3M, 6M, 1A, 2A, 3A, 4A, 5A, 10A). Regions represent distinct oceanic regimes and are derived using k-means cluster analysis (Figure 5). Specific locations are shown in Figure S6. Each column represents the decomposed terms for advection for the specified region. Each panel sorts the covariance ratio for each term by integration depth along the vertical axis and time aggregation scale along the horizontal axis. 